\title{
Relativistic Mass Ejecta from Phase-transition-induced Collapse of Neutron Stars
}

\author{
K. S. Cheng* and T. Harko \\ Department of Physics and Center for Theoretical and Computational Physics, \\ The University of Hong Kong, Pok Fu Lam Road, Hong Kong \\ Y. F. Huang \\ Department of Astronomy, Nanjing University, Nanjing, China \\ L. M. Lin \\ Department of Physics and Institute of Theoretical Physics, \\ The Chinese University of Hong Kong, Hong Kong, China \\ W. M. Suen \\ Department of Physics and Institute of Theoretical Physics, \\ The Chinese University of Hong Kong, Hong Kong, China and \\ McDonnell Center for the Space Sciences, \\ Department of Physics, Washington University, St. Louis, USA \\ X. L. Tian \\ Department of Physics, The University of Hong Kong, Pok Fu Lam Road, Hong Kong
}

(Dated: August 29, 2021) 


\begin{abstract}
We study the dynamical evolution of a phase-transition-induced collapse neutron star to a hybrid star, which consists of a mixture of hadronic matter and strange quark matter. The collapse is triggered by a sudden change of equation of state, which result in a large amplitude stellar oscillation. The evolution of the system is simulated by using a 3D Newtonian hydrodynamic code with a high resolution shock capture scheme. We find that both the temperature and the density at the neutrinosphere are oscillating with acoustic frequency. However, they are nearly $180^{\circ}$ out of phase. Consequently, extremely intense, pulsating neutrino/antineutrino fluxes will be emitted periodically. Since the energy and density of neutrinos at the peaks of the pulsating fluxes are much higher than the non-oscillating case, the electron/positron pair creation rate can be enhanced dramatically. Some mass layers on the stellar surface can be ejected by absorbing energy of neutrinos and pairs. These mass ejecta can be further accelerated to relativistic speeds by absorbing electron/positron pairs, created by the neutrino and antineutrino annihilation outside the stellar surface. The possible connection between this process and the cosmological Gamma-ray Bursts is discussed.
\end{abstract}

Keywords: dense matter : stars: neutron stars : stellar oscillations : phase transition- quark stars : gamma ray bursts.

*Electronic address: hrspksc@hkucc.hku.hk 


\section{INTRODUCTION}

Gamma-ray bursts (GRBs) are cosmic gamma ray emissions with typical fluxes of the order of $10^{-5}$ to $5 \times 10^{-4} \mathrm{ergs}^{-2}$ with the rise time as low as $10^{-4} \mathrm{~s}$ and the duration of bursts from $10^{-2}$ to $10^{3} \mathrm{~s}$. The distribution of the bursts is isotropic and they are believed to have a cosmological origin, with the observations showing that GRBs originate at extragalactic distances. The large inferred distances imply isotropic energy losses as large as $3 \times 10^{53}$ ergs for GRB 971214 and $3.4 \times 10^{54} \mathrm{ergs}$ for GRB 990123. For detailed reviews of GRBs properties see [1] and [2], respectively. The widely accepted interpretation of the phenomenology of GRBs is that the observable effects are due to the dissipation of the kinetic energy of a relativistically expanding fireball, whose primal cause is not yet known [1].

One fundamental question related to GRBs is how many intrinsically different categories they have. Each type may correspond to an intrinsically different type of progenitor, as well as to a different type of central engine. From the GRB sample collected by BATSE, a clear bimodal distribution of bursts was identified [2, 3]. Two criteria have been used to classify the bursts. The primary criterion is duration, and a separation line of $2 \mathrm{~s}$ has been adopted to separate the double-hump duration distribution of the BATSE bursts. The second criterion is the hardness-usually denoted as the hardness ratio within the two energy bands of the detector. On average, short GRBs are harder, while long GRBs are softer. Hence the observations show the existence of two classes of GRBs, long-soft, and shorthard, respectively. The short GRBs are hard because of the harder low-energy spectral index of the GRB spectral function [4]. More interestingly, short GRB spectra are broadly similar to those of long GRBs, if only the first 2 seconds of data of the long GRBs are taken into account. Afterglows observation shed some light into the physical nature of these two types of GRBs. The host galaxies of long GRBs are exclusively star forming galaxies, predominantly irregular dwarf galaxies [5]. The observations suggest that most, if not all of the long GRBs are produced during the core collapse of massive stars, called collapsars, as has been also suggested theoretically [6, 7, 8]. Both the observations and the theoretical models also support the idea that long GRBs are associated with supernova explosions [9].

The observations of SWIFT and of HETE led to a very different picture for the short GRBs [1, 2]. The basic result of all these observations is that short GRBs are intrinsically 
different from the long GRBs. Most of the short GRBs are found at the outskirts of elliptical galaxies, where the star forming rate is very low. Even if they can be associated with star forming regions, they are rather far away from the active zones [10]. In several cases a robust host galaxy has not been identified, but the host galaxy is of early type. Deep supernova searches have been performed, but with negative results [2]. This suggests that short GRBs may be associated with mergers of compact objects, like neutron star-neutron star or neutron star-black hole mergers, white-dwarf-neutron star or black hole mergers etc. [11, 12, 13, 14, 15].

The possibility that the conversion of neutron stars to strange quark stars may be the energy source for the cosmological gamma ray bursts was suggested in [16]. Neutron stars in low-mass X-ray binaries can accrete sufficient mass to undergo a phase transition to become strange stars. At the moment of its birth the strange star is very hot, with an interior temperature of around $\sim 10^{11} \mathrm{~K}$. By approximating the strange matter by a free Fermi gas, the thermal energy of the star is $E_{t h} \approx 5 \times 10^{51}\left(\rho / \rho_{0}\right)^{2 / 3} R_{6}^{3} T_{11}^{2}$ ergs, where $\rho$ is the average mass density, $\rho_{0}=2.8 \times 10^{14} \mathrm{~g} / \mathrm{cm}^{3}$ is the nuclear density, $R_{6}$ is the radius of the star in units of $10^{6} \mathrm{~cm}$, and $T_{11}$ is the temperature in units of $10^{11} \mathrm{~K}$. For $\rho=8 \rho_{0}, R_{6}=1, T_{11}=1.5$ the thermal energy of the newly formed quark star is $E_{t h} \approx 5 \times 10^{52} \mathrm{ergs}$. In the original model of [16] it was assumed that the star would cool by emission of neutrinos and antineutrinos, and that the neutrino pair annihilation process $\nu \bar{\nu} \rightarrow e^{-} e^{+}$operates near the strange star surface. The energy deposited due to this process is $E_{1} \approx 2 \times 10^{48}\left(T_{0} / 10^{11} K\right)^{4} \mathrm{ergs} \approx 10^{49}$ ergs, where $T_{0}$ is the initial temperature. The time scale for the deposition is around $1 \mathrm{~s}$. On the other hand, the processes $n+\nu_{e} \rightarrow p+e^{-}$and $p+\bar{\nu}_{e} \rightarrow n+e^{+}$play an important role in the energy deposition. The integrated neutrino optical depth due to all these processes is $\tau \approx 4.5 \times 10^{-2} \rho_{11}^{4 / 3} T_{11}^{2}$, where $\rho_{11}$ is the mass density in units of $10^{11} \mathrm{~g} / \mathrm{cm}^{3}$. The deposition energy can be estimated to be $E_{2} \approx E_{t h}\left(1-e^{-\tau}\right) \approx 2 \times 10^{52}$ ergs. Here the value of the neutron drip density, $\rho_{11}=4.3$ has been used, and it has been assumed that all the thermal energy of the star is lost in neutrinos. The process $\gamma \gamma \rightarrow e^{-} e^{+}$inevitably leads to the creation of a fireball, and this fireball will expand outward. The expanding shell interacts with the surrounding interstellar medium, and its kinetic energy is radiated through nonthermal processes in shocks. However, this model did not consider any internal dynamics, e.g. heat transport, viscous damping, shock dissipation etc, which can affect the neutrino emission dramatically. In fact the numerical simulations indicate that the temperature on 
the neutrinosphere is rapidly changing with time, steady neutrino emission is not possible.

The presence of oscillations of the resulting quark star produced by the phase transition induced collapse of a neutron star is one of the most intriguing features of the simulations performed with our Newtonian numerical code introduced in [17]. Recently, the collapse process with a conformally flat approximation to general relativity was also simulated in [18]. The works of [17, 18] focus on the gravitational wave signals emitted by the collapse process.

It is the purpose of the present paper to consider another important implication of this result, namely, the effect of the oscillations of the newly formed quark star on the neutrino emission. The oscillations can enhance the neutrino emission rate in a pulsating manner, and the neutrinos are emitted in a much shorter time scale. Therefore the neutron-quark phase transition in compact objects may be the energy source of GRBs.

This paper is organized as follows. The phase transition process from neutron stars to hybrid stars, which consists of a mixture of strange quark matter and hadronic matter, is summarized in Section II. We describe our numerical code, which is used to simulate the dynamical evolution of star after phase transition in Section III. In Section IV, we calculate the neutrino and antineutrino emission from the neutrinosphere and the electron/positron pair creation rate due to neutrino and antineutrino annihilation process. In Section $\mathrm{V}$, we calculate mass ejection from stellar surface by absorbing neutrinos/pairs and their subsequent acceleration by the pairs outside the star. In Section VI we apply our model to GRBs. Finally a brief summary and discussion is presented in Section VII.

\section{DESCRIPTION OF THE PHASE TRANSITION}

The quark structure of the nucleons, suggested by quantum chromodynamics, indicates the possibility of a hadron-quark phase transition at high densities and/or temperatures, as suggested by [19, 20, 21]. Theories of the strong interaction, like, for example, the quark bag models, suppose that breaking of physical vacuum takes place inside hadrons. If the hypothesis of the quark matter is true, then some of neutron stars could actually be strange stars, built entirely of strange matter [22, 23, 31, 32, 33].

The central density of compact stellar objects may reach values of up to ten times nuclearmatter saturation density, and therefore a phase transition to deconfined quark matter, or 
pion and kaon condensates, should take place at least in the central region of the neutron stars. In the case of the transition to quark matter, in addition to a phase of unpaired normal quark matter, present at low densities, several superconducting phases, such as the twoflavor color superconductor (2SC) phase or the gapless Color-flavor-lock (CFL) phase can also occur at the large baryon densities reached at the central regions of a compact star. The transition from the hadronic to the quark phase could proceed in two steps. In the first step, a transition from hadronic matter to normal quark matter or to a 2SC phase takes place, due to the increase of the baryonic density at the center of the star. This increase in density may be due to mass accretion from the fall-back material or rapidly spin-down of the star. The newly formed hybrid or quark star, containing some 2SC quark matter, can become meta-stable, and decay into a star containing a CFL phase [24]. Pure hadronic compact stars above a threshold value of their mass are metastable. The metastability of hadronic stars originates from the finite size effects in the formation process of the first strange quark matter drop in the hadronic environment [25].

A phase transition between the hadronic and quark phase occurs when the pressures and the chemical potentials in the two phases are equal, $P_{h}=P_{q}, \mu_{h}=\mu_{q}$, where $P_{h}, \mu_{h}$ and $P_{q}$, $\mu_{q}$ are the pressures and the chemical potentials in the hadron and quark phase, respectively. In the present Section, unless otherwise explicitly specified, we use the natural system of units with $\hbar=c=1$. If the transition pressure is less than that existing at the center of the compact object, the transition can occur. For finite values of the surface tension, complicated structures can develop in the mixed phase, such as drops, rods, and slabs [28]. The formation of a mixed phase is the result of two competing processes: the size of the barrier that the system has to overcome in order to form a structure, and the size of the perturbation of the system. One method to see if the phase transition can proceed or not is to compare the temperature reached by the system immediately after the conversion with the height of the barrier. If the temperature is not much lower than the height of the barrier, the structure formation proceeds thermally, and it is very rapid [29, 30]. If the temperature is low, new structures can form only via quantum nucleation, which is a very slow process [24].

The thermal nucleation rate of quark drops can be estimated in the framework of the nucleation theory as $R_{n u c l} \approx a \exp \left(-W_{c} / T\right)$, where the prefactor $a$ (the product of the dynamical prefactor and of the statistical prefactor) is the prod- 
uct of a density and a growth factor, $W_{c}=W\left(R_{c}\right)$ represents the work needed to form the smallest drop capable of growing, and $T$ is the temperature. $W_{c}$ corresponds to the maximum of the free energy of the drop in the new phase. The dynamical prefactor for the nucleation rate of bubbles or droplets in first-order phase transitions for the case where both viscous damping and thermal dissipation are significant has been obtained in [26]. This formalism was applied for the study of the nucleation of quark-gluon plasma from hadronic matter in [27]. By taking into account the explicit forms of the temperature dependent quark matter equation of state, of the dissipative factors and of the statistical prefactor, one obtains a complicated dependence of the prefactor on the temperature. However, the prefactor in this model is based on poorly known physical parameters (like, for example, the shear viscosity of the hadronic phase). In order to obtain some qualitative estimates of the nucleation rate we use the thermodynamical approach developed in [29, 30]. The form of the prefactor can be obtained from general thermodynamical considerations as $a \approx T^{4}$, and it is very little dependent on some particular choices (for example in the low temperature limit the prefactor in the expression for $R_{\text {nucl }}$ can be replaced by the baryon chemical potential). This form for the prefactor does not include any kinematics, e.g. the microscopic processes required to transform a gas of hadrons into a gas of quarks. In the expression of the nucleation rate the dominant term is the exponential. The free energy is given by $W=-4 \pi R^{3} \Delta P / 3+4 \pi \sigma R^{2}+8 \pi \gamma R+N_{q} \Delta \mu$, where $\Delta P=P_{q}-P_{h}$ is the pressure difference, $\sigma=\sigma_{q}+\sigma_{h}$ is the surface tension, $\gamma=\gamma_{q}-\gamma_{h}$ is the curvature energy density and $\Delta \mu=\mu_{q}-\mu_{h}$ is the difference in the chemical potential. $N_{q}$ is the total baryon number in the quark drop [24, 29, 30, 34].

The free energy has a maximum at the critical radius $R_{c}=\sigma(1+\sqrt{1+b}) / C$, where $C=\Delta P-n_{q} \Delta \mu$ and $b=2 \gamma C / \sigma^{2}$. The corresponding free energy is

$$
W_{c}=8 \pi \sigma^{3}\left[1+(1+b)^{3 / 2}+3 b / 2\right] / 3 C^{2} .
$$

The number $N$ of drops of the new phase formed inside the old phase in a volume $V$ in a time $t$ is given by $N=R_{\text {nucl }} V t$. Let $\lambda$ be the spacing between two drops in the mixed phase. The number of drops in a volume $V$ is given by $V / \lambda^{3}$. The number of drops produced while the front moves over a distance $\lambda$ must be of the order of the drops that are present in the 
mixed phase, $R_{n u c l} V t=R_{n u c l} S(\lambda / v) \geq V / \lambda^{3}=S / \lambda^{2}$, where $v$ is the velocity of the front and $S$ its surface area. Therefore in order for thermal nucleation take place the condition $W_{c} / T \leq \ln \left(T^{4} \lambda^{4} / v\right)$ must be satisfied. The process of absorption of a hadron into a pure quark matter phase can also be described phenomenologically as the fusion of a small drop of quarks growing into a much larger drop.

The nature of the conversion process from the neutron to the quark matter is not yet fully understood, and there is no clear theoretical evidence if it is a deflagration or a detonation. Indeed, for realistic equations of state of quark and neutron matter and when matter is assumed to be in $\beta$ equilibrium, detonation is difficult to achieve $[35,36]$. However, it would be still possible to obtain a detonation if the matter immediately after the front is not yet in $\beta$-equilibrium [24]. It is also important to note that neutrino trapping delays $\beta$ stability. According to the analysis of [24], when the drop starts expanding, the process of conversion can be extremely fast, within the layer in which deconfinement is energetically favorable, even in the absence of the weak processes. In this case, the conversion front moves at the velocity of the deflagration front $v_{d f}$, which approaches the velocity of sound. As the conversion layer moves outward, the front enters the region of mixed phase, where $v_{d f}$ decreases until it vanishes at the low density boundary of the mixed phase.

If the conversion is indeed extremely fast, taking place at speeds close to the speed of sound, then the typical time scale for the transition can be estimated as $\tau_{t r}=R / c_{s}$, where $R$ is the radius of the neutron star and $c_{s}$ is the speed of the sound. A simple phenomenological model for the evolution of the quark phase can be obtained by assuming the relation $d r / d t=\left(r-R_{c}\right) / \tau_{t r}$ [34], which gives for the transition time scale $T_{t r}$ from a microscopic quark drop to a quark matter distribution of a macroscopic size the expression

$$
T_{t r} \approx 10^{-4} N_{q}^{-1 / 3} R_{6} \ln \frac{R}{R_{q}} \mathrm{~s},
$$

where $R_{6}$ is the neutron star radius in units of $10^{6} \mathrm{~cm}, R_{q} \sim 300 R_{c}$ is the initial size of the quark drop [34], and $N_{q}$, which could be as large as $10^{48}$ [37], is the number of quark drops inside the core of the neutron star. In this case $R$ in Eq. (2) is replaced by $R / N_{q}^{1 / 3}$. Therefore the phase transition may take place in a time scale much shorter than submillisecond. 


\section{SIMULATION OF THE PHASE-TRANSITION INDUCED COLLAPSE}

In this paper we focus on studying the phase-transition induced collapse of neutron star to a hybrid quark star, which consists of a mixture of strange quark matter and hadronic matter, and want to demonstrate that this process can produce relativistic ejecta, which could be a mechanism for GRBs. In order to avoid other complications we would like to simulate a non-rotating, phase-induced collapse of a compact object, with a mixed phase of quark matter and nuclear matter. In this model gravitational radiation will not be emitted. Hence we can focus on studying the neutrino and pair emissions and subsequently gamma-ray emission via interaction between the interstellar medium from this system. In our simulations, we will not simulate the phase transition process. Instead, we assume that a fast phase transition has happened (e.g., via a detonation mode) so that the initial neutron star has converted to a quark star in a timescale shorter than the dynamical timescale of the system. We assume that the normal matter inside the initial neutron star has suddenly changed to quark matter at $t=0$. This is achieved by changing the EOS at $t=0$ after the initial hydrostatic equilibrium neutron star has been constructed. We then simulate the resulting dynamics of the system triggered by the collapse.

\section{A. Description of the numerical code}

Our numerical code is based on the three-dimensional numerical simulation in Newtonian hydrodynamics and gravity. The quark matter of the mixed phase is described by the MIT bag model and the normal nuclear matter is described by an ideal fluid EOS. This code has been used to study the gravitational wave emission from the phase-induced collapse of the

neutron stars [17]. Here we briefly summarize the main equations and the numerical scheme involved. A detailed discussion can be found in [17].

The system of equations describing the non-viscous Newtonian fluid flow is given by

$$
\begin{gathered}
\frac{\partial \rho}{\partial t}+\nabla \cdot(\rho \mathbf{v})=0 \\
\frac{\partial}{\partial t}\left(\rho v_{i}\right)+\nabla \cdot\left(\rho v_{i} \mathbf{v}\right)+\frac{\partial P}{\partial x_{i}}=-\rho \frac{\partial \Phi}{\partial x_{i}} \\
\frac{\partial \tau}{\partial t}+\nabla \cdot((\tau+P) \mathbf{v})=-\rho \mathbf{v} \cdot \nabla \Phi
\end{gathered}
$$


where $\rho$ is the mass density of the fluid, $\mathbf{v}$ is the velocity with Cartesian components $v_{i}$ $(i=1,2,3), P$ is the fluid pressure, $\Phi$ is the Newtonian potential and $\tau$ is the total energy density, $\tau=\rho \epsilon+\rho \mathbf{v}^{2} / 2$, and $\epsilon$ is the internal energy per unit mass of the fluid, respectively. The Newtonian potential $\Phi$ is obtained by solving the Poisson equation, $\nabla^{2} \Phi=4 \pi G \rho$. The system is completed by specifying an equation of state $P=P(\rho, \epsilon)$.

The above hydrodynamics Eqs. (3)-(15) can be rewritten in the so-called flux-conservative form, which can be solved numerically using quite standard high resolution shock capturing (HRSC) schemes. A HRSC scheme, using either exact or approximate Riemann solvers, with the characteristic fields (eigenvalues) of the system, obtains the solution of a local Riemann problem at every cell interface of a finite-differencing grid. Such schemes have the ability to resolve discontinuities in the solution (e.g., shock waves) by construction. Moreover, they have high accuracy in regions where the fluid flow is smooth. In general, integrating the hydrodynamics equations by a HRSC scheme involves the choices of an appropriate numerical flux formula and a reconstruction method of the state variables $(\rho, \epsilon, \mathbf{v})$ for solving the Riemann problems at the cell interfaces. In our code, we use the Roe's approximate Riemann solver [38] for the numerical fluxes and the third-order piecewise parabolic method [39] for the reconstruction. For the temporal discretization, we use a basic two-step method to achieve second-order accuracy in time.

In the simulations, we introduced a very low density atmosphere outside the star. The "artificial" atmosphere is not physical, but it is important for the stability of the hydrodynamical code. This is due to the problem that the hydrodynamical codes cannot in general handle vacuum regions where the density is zero. In order to avoid a significant influence of the atmosphere on the dynamics of the physical system, it is necessary to choose the density of the atmosphere $\rho_{\text {atm }}$ to be much smaller than the density scale of interest. For the results reported in Section 3.4, we set $\rho_{\text {atm }}$ to be $3 \times 10^{9} \mathrm{~g} / \mathrm{cm}^{3}$. The effects of different atmospherical values have been compared in [40].

\section{B. Equation of state}

The equation of state (EOS) for neutron stars is highly uncertain. We could try all possible existing realistic EOS in our study. However, the main purpose of this paper is to demonstrate that during the phase-transition induced collapse of a neutron star extremely 
intense, pulsating and very high energy neutrinos can be emitted. The effect is governed mainly by the amount of pressure reduction after the phase transition as compared to the initial neutron star model. For simplicity we will use a polytropic EOS for the initial equilibrium neutron star:

$$
P=k_{0} \rho^{\Gamma_{0}},
$$

where $k_{0}$ and $\Gamma_{0}$ are constants. On the initial time slice, we also need to specify the specific internal energy $\epsilon$. For the polytropic EOS, the thermodynamically consistent $\epsilon$ is given by

$$
\epsilon=\frac{k_{0}}{\Gamma_{0}-1} \rho^{\Gamma_{0}-1} .
$$

Note that the pressure in Eq. (6) can also be written as

$$
P=\left(\Gamma_{0}-1\right) \rho \epsilon .
$$

In order to describe the physical properties of the neutron stars after the phase transition, we consider that the star can be divided in three regions. At the center of the star, where $\rho>\rho_{q}$, we have a pure quark core (Region I), which is described by the MIT bag model equation of state, so that the pressure $P$ is given by

$$
P=P_{\mathrm{q}}=\frac{1}{3}(\rho+\rho \epsilon-4 B), \rho>\rho_{q},
$$

where $B$ is the bag constant, and $\rho_{q}$ is a critical density for which all the hadrons are deconfined into quarks. It should be noticed that $P_{q}$ is not in the usual form of $P=\left(\rho_{\text {tot }}-4 B\right) / 3$, where $\rho_{\text {tot }}$ is the (rest frame) total energy density. It is because in our Newtonian simulations, we use the rest mass density $\rho$ and specific internal energy $\epsilon$ as fundamental variables in the hydrodynamics equations. We assume that the quark core is absolutely stable. The quark core is surrounded by a mixed phase of quark and nuclear matter (Region II) that can exist if the density of the region is higher than a certain critical value $\rho_{t r}$ (quark seeds can spontaneously produce everywhere when $\rho \geq \rho_{t r}$ ). Explicitly, the pressure in the mixed phase is given by

$$
P=\alpha P_{\mathrm{q}}+(1-\alpha) P_{\mathrm{n}}, \rho_{q}>\rho>\rho_{t r}
$$

where

$$
P_{\mathrm{n}}=\left(\Gamma_{n}-1\right) \rho \epsilon,
$$


and

$$
\alpha=\left\{\begin{array}{cc}
\left(\rho-\rho_{t r}\right) /\left(\rho_{q}-\rho_{t r}\right), & \text { for } \rho_{t r}<\rho<\rho_{\mathrm{q}}, \\
1, & \text { for } \rho_{q}<\rho,
\end{array}\right.
$$

is defined to be the scale factor of the mixed phase [17]. $\Gamma_{n}$ is not necessarily equal to $\Gamma_{0}$. Finally, we have a normal nuclear matter region (Region III), extending from $\rho<\rho_{t r}$ to the surface of the star, so that in this region

$$
P=P_{\mathrm{n}}, \text { for } \rho \leq \rho_{t r}
$$

A more detailed discussion about such hybrid quark stars can be found in [17].

The total energy density $\rho_{\text {tot }}$, which includes the rest mass contribution, is decomposed as $\rho_{\text {tot }}=\rho+\rho \epsilon$. We choose $\Gamma_{n}<\Gamma_{0}$ in our simulations to take into account the possibility that the nuclear matter may not be stable during the phase transition process, and hence some quark seeds could appear inside the nuclear matter, or the convection, which can occur during the phase transition process, can mix some quark matter with the nuclear matter. In the presence of the quark seeds in the nuclear matter, the effective adiabatic index will be reduced. The possible values of $B^{1 / 4}$ range from $145 \mathrm{MeV}$ to $190 \mathrm{MeV}$ [41, 42, 43, 44]. For $\rho>\rho_{q}$, the quarks will be deconfined from nucleons. The value of $\rho_{q}$ is model dependent; it could range from 4 to $8 \rho_{\text {nuc }}$ [44, 45, 46], where $\rho_{\text {nuc }}=2.8 \times 10^{14} \mathrm{~g} \mathrm{~cm}^{-3}$ is the nuclear density.

There are two issues regarding our EOS model to be addressed: (1) It should be noted that, for simplicity, we do not include the change in the internal energy from the phase transition when setting the initial data for the collapse. The binding energy released in the phase transition effectively leads to a slightly harder EOS due to the thermal pressure. This could be modeled by using a larger value of $\Gamma_{n}$ (comparing to the one we used in this work). However, as long as the EOS after the phase transition is softer than that of the initial neutron star, the star will still collapse and stellar pulsations will be triggered. (2) Furthermore, the parameter $\rho_{t r}$ in our EOS model should be considered as the density below which the matter is dominated by hadronic matter. It is noted that strange quark matter (if it is more stable) cannot convert back to hadronic matter by decreasing the density. However, when a fluid element originally in the mixed phase moves to the lower density region $\left(\rho<\rho_{t r}\right)$, the fluid element will mix with a large amount of hadronic matter. Hence, 


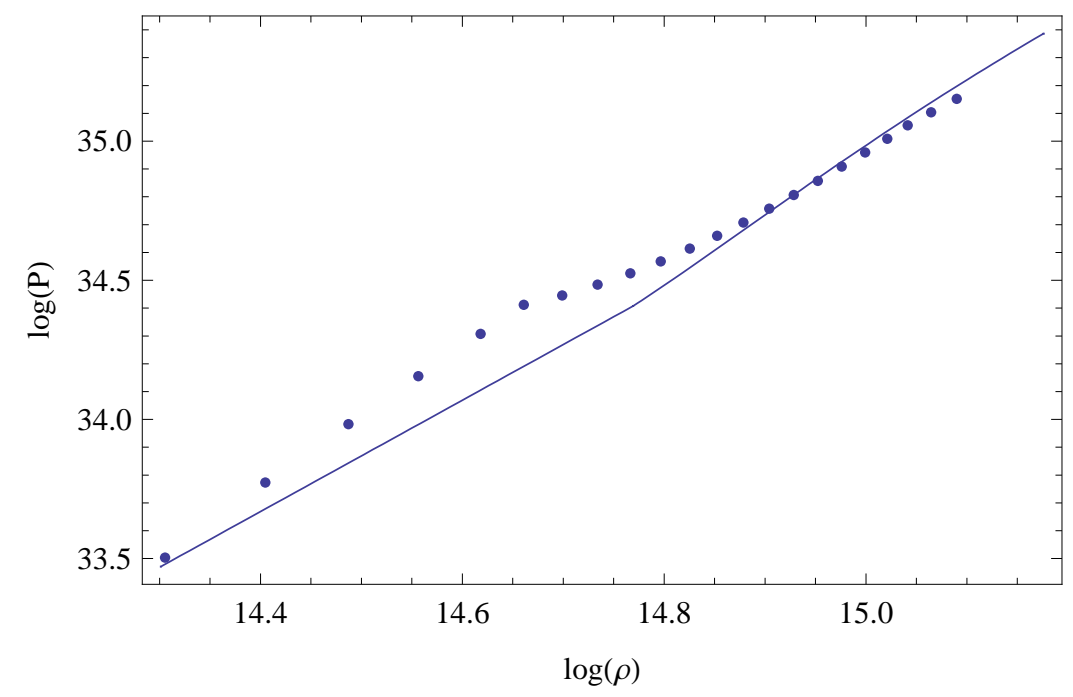

FIG. 1: Comparison of the equation of state of the mixed quark-hadron phase proposed in [28] for $K=240 \mathrm{MeV}$ and effective mass $m * / m=0.78$ (dotted curve), and Eq. (10), the equation of state if the mixed phase used in the present simulations (solid curve).

we approximate that the pressure in the outer region of the star is dominated by the hadronic part, which is modeled by an ideal gas.

In the simulations, we choose $\Gamma_{0}=2, \Gamma_{n}=1.85, B^{1 / 4}=160 \mathrm{MeV}$ and $\rho_{q}=9 \rho_{\text {nuc }}$. The transition density $\rho_{t r}$ is defined to be at the point where $P_{q}$ vanishes initially.

In Eq. (10), we have used a very simple linear combination of strange quark matter EOS and hadronic matter to represent the EOS of mixed phase, which consists of a mixture of strange quark drops and hadronic matter. In fact, the properties of a mixed quark-hadron phase and its implications for hybrid star structure were considered in [28]. In Fig. 11 we compare the EOS of the mixed phase obtained in [28] for a compression modulus $K=240$ $\mathrm{MeV}$ and an effective mass at saturation density of $m * / m=0.78$ with Eq. (10) used in the present simulation. We can see that these EOSs are quite close to each other. Although we use Eq. (10) in our simulation solely based on simplicity, we believe that the simulation results will not be changed qualitatively if we replace Eq. (101) by the EOS given in [28].

In the mixed phase we have assumed that the effective bag constant $B$ is a linearly dependent function of the density. The equation of state used by us reproduces quite well the EOS proposed in [28] to describe the mixed quarkhadron phase. It is important to note that the main purpose of our paper is to focus on the study of the dynamical response of the star after a sudden 
phase transition, no matter what the fine details of the phase transition are. A full and exact description of the phase transition would require a very precise knowledge of the physical parameters describing both quark and hadronic matter. However, taking into account the uncertainties in our present knowledge of the properties of matter at high densities, our investigations could certainly give at least a qualitative picture of the astrophysical implications of hadron-quark phase transitions in compact stars.

\section{The Neutrinosphere}

For a new born compact star, the internal temperature is so high that neutrinos will be trapped inside the star for at least a few seconds (cf. [47] for a general review). However, neutrinos very near the surface of the star can still escape from the star because the optical depth near the stellar surface is low. Quantitatively we can define a surface called the neutrinosphere with a radius $R_{\nu}$ as follows, e.g. [47, 48],

$$
\tau_{e f f}=\int_{R_{\nu}}^{\infty} d r \kappa_{e f f}(r)=1
$$

where the effective optical depth, $\tau_{e f f}$ is defined as the inverse mean free path and the effective opacity, $\kappa_{\text {eff }}$ is given by

$$
\left\langle\kappa_{e f f}\right\rangle(r)=1.202 \times 10^{-7} \rho_{10}(r)\left(\frac{T_{\nu}}{4 \mathrm{MeV}}\right)^{2} \frac{1}{\mathrm{~cm}} .
$$

It is clear that this surface is a function of the temperature and of the density.

\section{Simulation Results}

The total time span of the simulations is $\sim 5 \mathrm{~ms}$, and the time step is $3.7 \times 10^{-4} \mathrm{~ms}$. For all the simulations we report in this paper, the grid spacing is set to be $d x=0.28 \mathrm{~km}$ and the outer boundary of the computational domain is at $27.5 \mathrm{~km}$, which is about two times the stellar radius of our models. With the grid resolution we used for the simulations, we see that numerical damping becomes important after about $3 \mathrm{~ms}$. We shall thus only present the numerical results up to $3 \mathrm{~ms}$. During the simulations, a low-density atmosphere is added

outside the neutron star. The density and temperature of the atmosphere are $3 \times 10^{9} \mathrm{~g} / \mathrm{cm}^{3}$ and $0.003 \mathrm{MeV}$ respectively. 

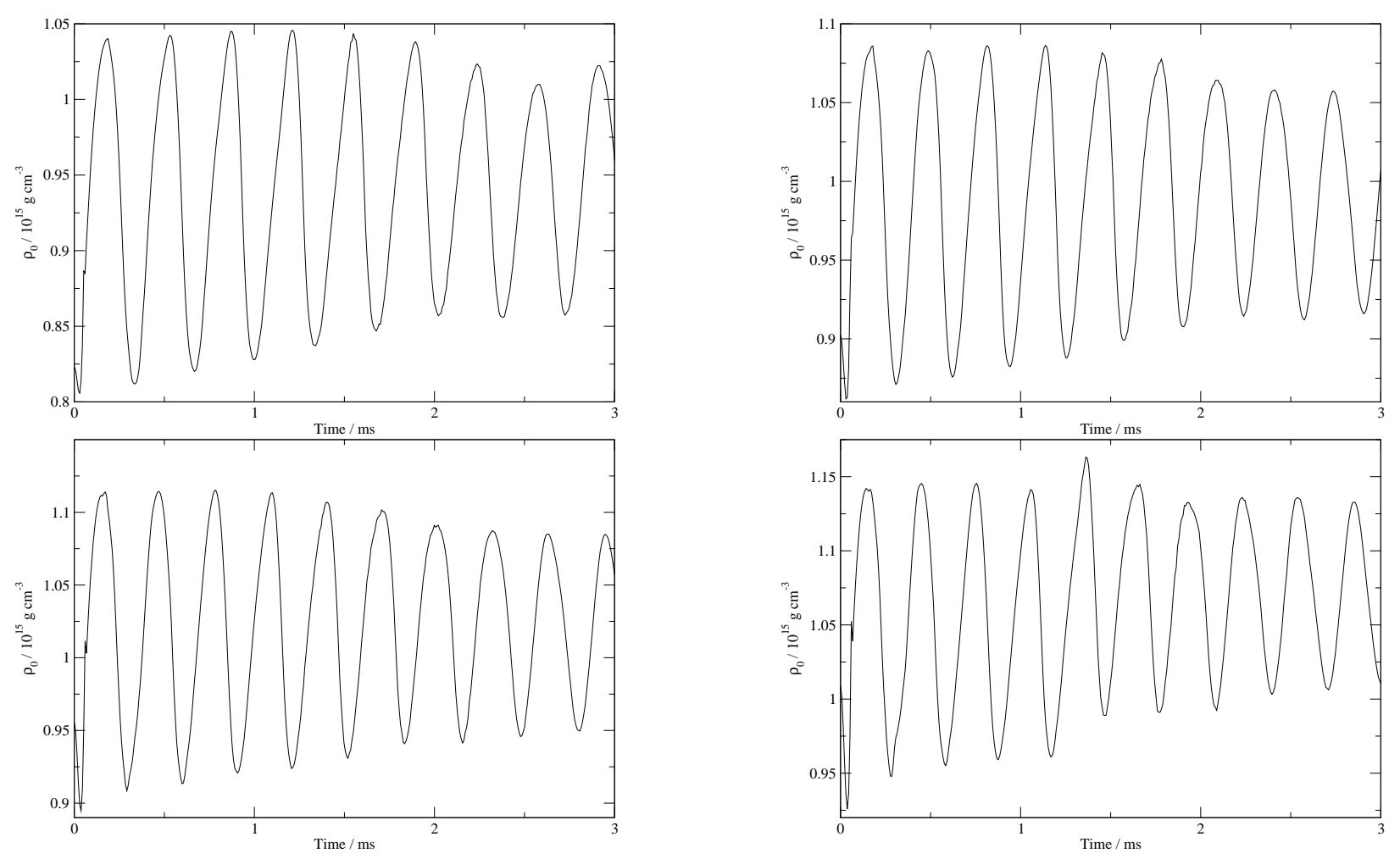

FIG. 2: Variation of the central density with respect to the time for $M=1.55 M_{\odot}$, (left upper figure), $M=1.7 M_{\odot}$, (right upper figure), $M=1.8 M_{\odot}$, (left lower figure), $M=1.9 M_{\odot}$, (right lower figure).

Fig. 2 shows the oscillations of the central density for stars with $M=1.55,1.7,1.8$ and $1.9 M_{\odot}$ respectively.

We can see that the oscillation period gradually decreases from $\sim 0.32 \mathrm{~ms}$ to $\sim 0.29 \mathrm{~ms}$, when the mass of the star increases. It is because the radial oscillation is basically acoustic oscillation (cf. Lin et al. 2006) therefore frequency essentially increases with the average density. In fact the frequency is roughly scaled according to $\rho_{0}^{1 / 2}$. Hence, the oscillation period is smaller for more massive stars because they have higher density. We can also see that the oscillation amplitudes decrease slightly after $2 \mathrm{~ms}$. We note that the damping is due partly to finite-differencing errors and physical effects, such as mass-shedding near the surface. One might worry that the large amplitude oscillations seen in the collapse simulations were numerical artifacts due to numerical errors or instability.

In order to demonstrate that the oscillations were in fact triggered by the phase transition, we show in Fig. [3 the evolution of the central density (normalized by its initial value) for an 


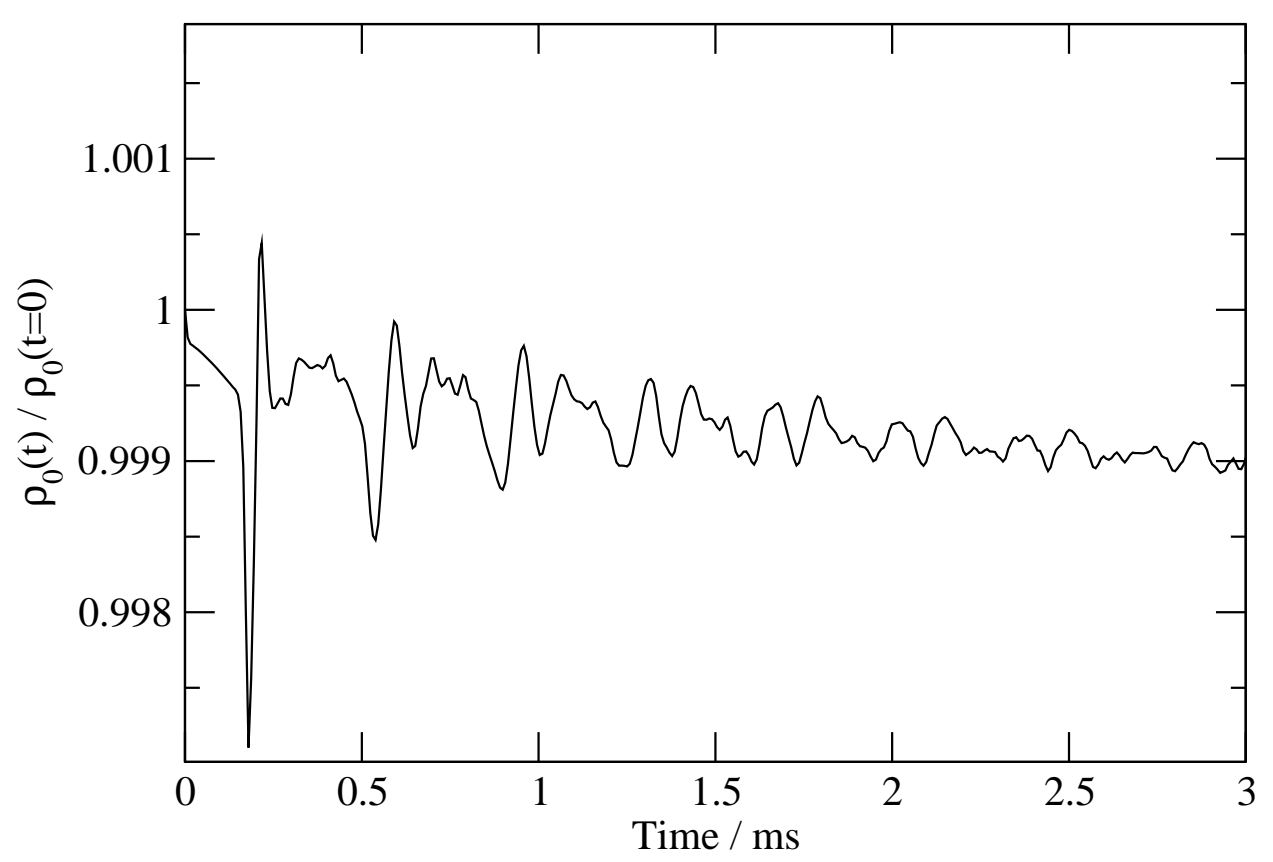

FIG. 3: Evolution of the central density (normalized by its initial value) for an unperturbed equilibrium neutron star with $M=1.8 M_{\odot}$.

unperturbed equilibrium neutron star model with $M=1.8 M_{\odot}$. We see that the amplitude of the oscillations of the star triggered by finite-differencing errors is much smaller than that seen in the collapse models. Furthermore, there is no obvious periodic features in Fig. 3,

With the simulated density and temperature profiles on a given time slice, we can calculate the position of the neutrinosphere $R_{\nu}$ (see Eq. (14)) with a trial-and-error method. Since $R_{\nu}$ is a function of both temperature and density (cf. previous Section), it also oscillates with the same period as the central density. Figs. 4 and Figs. 5 show the temperatures and the densities at the neutrinosphere as a function of time for neutron stars with masses of $1.55,1.7,1.8$ and $1.9 M_{\odot}$, respectively.

We can see that both the temperature and the density at $R_{\nu}$ are pulsating, with the same period as that of the central density, but they are almost $180^{\circ}$ out of phase to each other.

To show the origin of the pulse like temperature and density evolution, we pick 5 time slices from $t=0$ to $t=0.16 \mathrm{~ms}$, and we focus on the temperature and density evolution at the neutrinosphere. Figs. [6 show the time evolution of the temperature and of the density profile of a star with $1.55 M_{\odot}$ respectively.

We find that the core temperature is rising immediately after the phase transition, which starts at $t=0$, and the heat is moving outward. The neutrinosphere is moving inward 

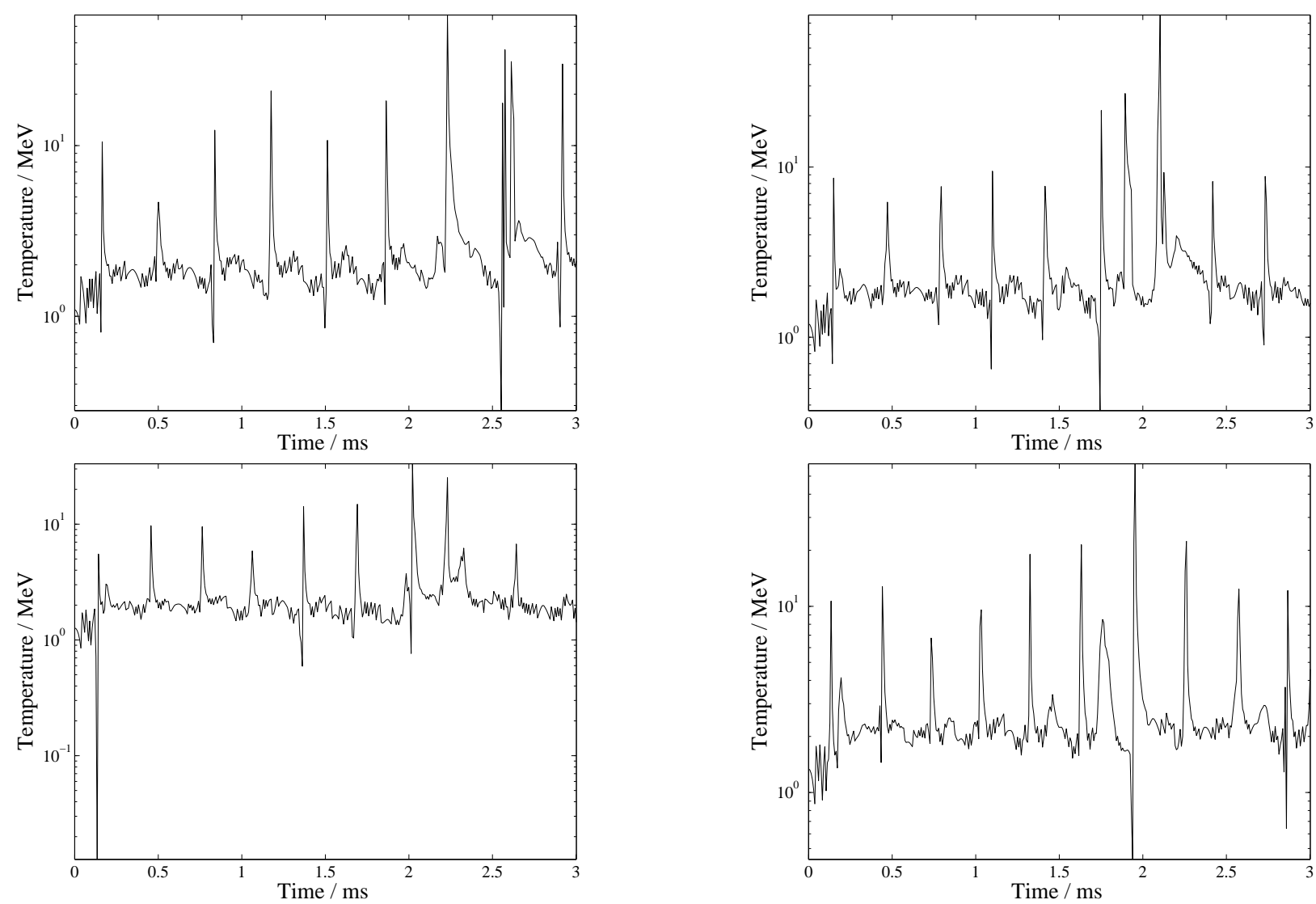

FIG. 4: Temperature at the neutrinosphere versus time for $M=1.55 M_{\odot}$, (left upper figure), $M=1.7 M_{\odot},\left(\right.$ right upper figure), $M=1.8 M_{\odot},\left(\right.$ left lower figure), $M=1.9 M_{\odot}$, (right lower figure).

because the matter is falling in. The star is shrinking until $t \sim 0.16 \mathrm{~ms}$, which is half of the oscillation period, and the density at the neutrinosphere is also at its minimum. Also at $t \sim 0.16 \mathrm{~ms}$, the outward heat pulse meets the infalling material density minimum. This can also be understood from the definition of the radius of the neutrinosphere, which is defined as the optical depth equal to unity. Therefore, when the temperature at the neutrinosphere is maximum, the corresponding density must be at the minimum value.

While the resolutions we used in the simulations (which are limited by the computational resource) are good enough to model the global dynamics of the star accurately, we note that this is not the case near the stellar surface where the density is very small and changing rapidly. In particular, the grid resolution near the neutrinosphere is not very high. We have compared the numerical results obtained with a few different resolutions in order to examine the effects of resolution. 

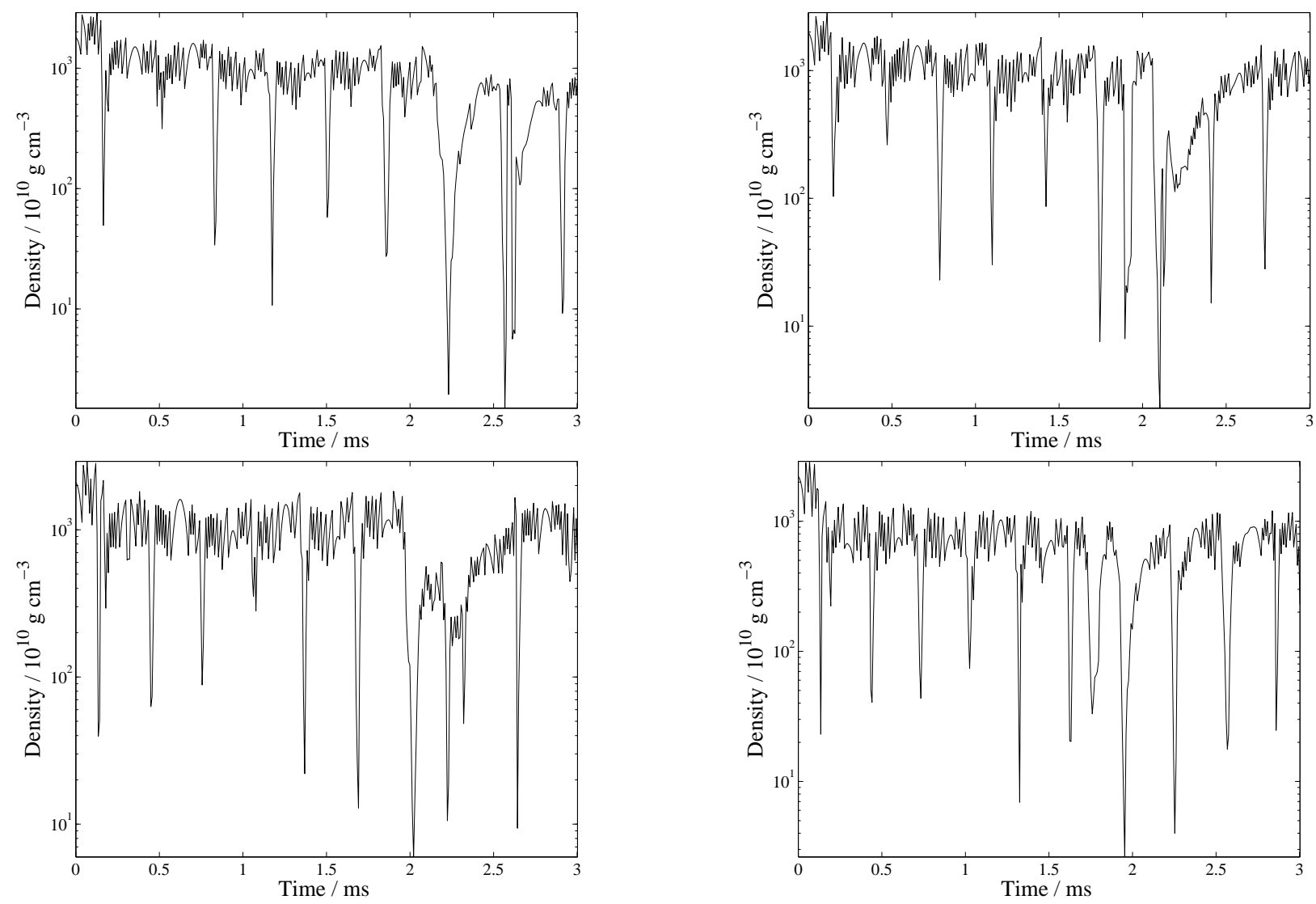

FIG. 5: Density at the neutrinosphere versus time for $M=1.55 M_{\odot}$, (left upper figure), $M=$ $1.7 M_{\odot},\left(\right.$ right upper figure), $M=1.8 M_{\odot}$, (left lower figure), $M=1.9 M_{\odot}$, (right lower figure).

In Figs. 7, we show the evolutions of the temperature and density at the neutrinosphere for the collapse model with $M=1.75 M_{\odot}$. The figures show that the different resolution results agree quite well qualitatively during about the first $1.5 \mathrm{~ms}$. In particular, the period of the pulses does not depend strongly on the resolution. The maximum variation in period for different grid size is given by $0.28 \mathrm{~km} / C_{s}$, where $C_{s} \sim 10^{9} \mathrm{~cm} / \mathrm{s}$ is sound speed near the surface, and it gives the maximum shift in period $\sim 0.03 \mathrm{~ms}$.

\section{EMISSION OF NEUTRINOS AND $e^{ \pm}$PAIRS}

\section{A. Neutrino Luminosity}

The neutrino luminosity is given by [49],

$$
L_{\nu}=4 \pi r^{2} c \frac{1}{2 \pi \hbar^{2}} \int \frac{E_{\nu} d^{3} \mathbf{p}_{\nu}}{1+\exp \left(E_{\nu}-\mu_{\nu} / k T_{\nu}\right)},
$$



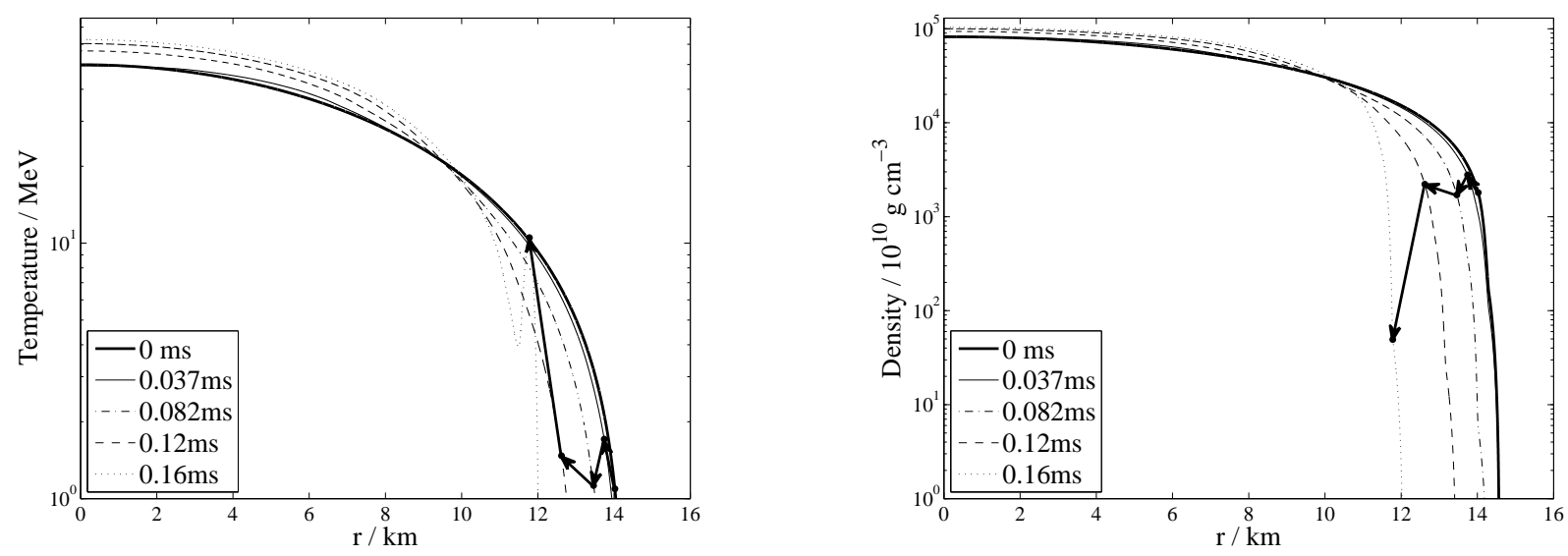

FIG. 6: Temperature evolution (figure on the left) and density evolution (figure on the right). The arrow indicates the position at which the temperature (density) change occurs at the neutrinosphere.
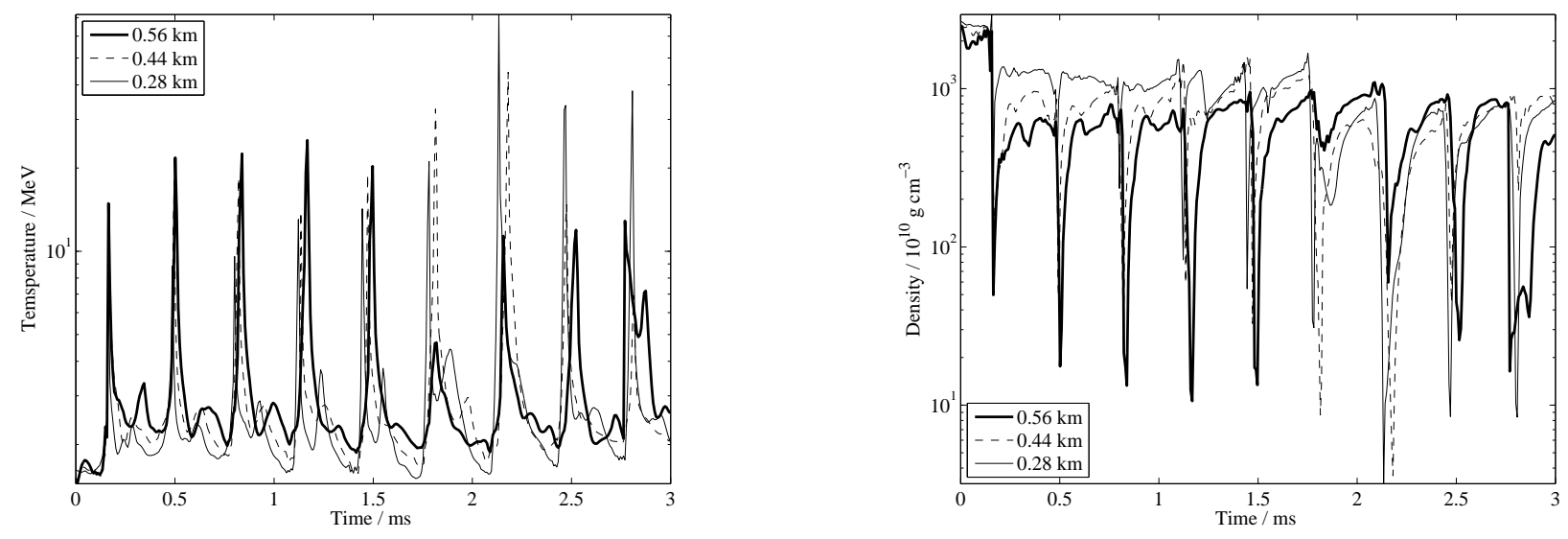

FIG. 7: Evolutions of the temperature (figure on the left) and density (figure on the right) at the neutrinosphere for $M=1.75 M_{\odot}$, with three different grid resolutions.

where $\mu_{\nu}$ is the neutrino chemical potential. Taking $\mu_{\nu}=0$, the neutrino luminosity emitted from the neutrinosphere is given by $L_{\nu}=7 \pi R_{\nu}^{2} a c T_{\nu}^{4} / 16$, where $a=4 \sigma / c$ is the radiation constant, $\sigma$ is the Stefan-Boltzmann constant, and $T_{\nu}$ is the temperature of the neutrinosphere. If we assume equal luminosities for neutrinos and antineutrinos, the combined luminosity for a single neutrino flavor is

$$
L_{\nu, \bar{\nu}}=L_{\nu}+L_{\bar{\nu}}=\frac{7}{8} \pi R_{\nu}^{2} a c T_{\nu}^{4}
$$

The effect of coherent forward scattering must be taken into account when considering 
the oscillations of neutrinos traveling through matter (author?) [50]. Although different flavor neutrinos have different $R_{\nu}$, yet they have approximately the same value of luminosity for all flavors [51, 52] (for general reviews and in depth discussions of the present status of neutrino oscillations and their astrophysical implications see [53, 54, 55, 56]). Therefore the total luminosity is around three times of a single neutrino flavor luminosity

$$
\begin{aligned}
L & =L_{\nu_{e}, \bar{\nu}_{e}}+L_{\nu_{\mu}, \bar{\nu}_{\mu}}+L_{\nu_{\tau}, \bar{\nu}_{\tau}} \\
& =\frac{21}{8} \pi R_{\nu}^{2} a c T_{\nu}^{4} .
\end{aligned}
$$

Using $R_{\nu}$ and $T_{\nu}$ obtained from the last Section, we compute the neutrino luminosity as a function of time. The results are shown in Fig. 8.
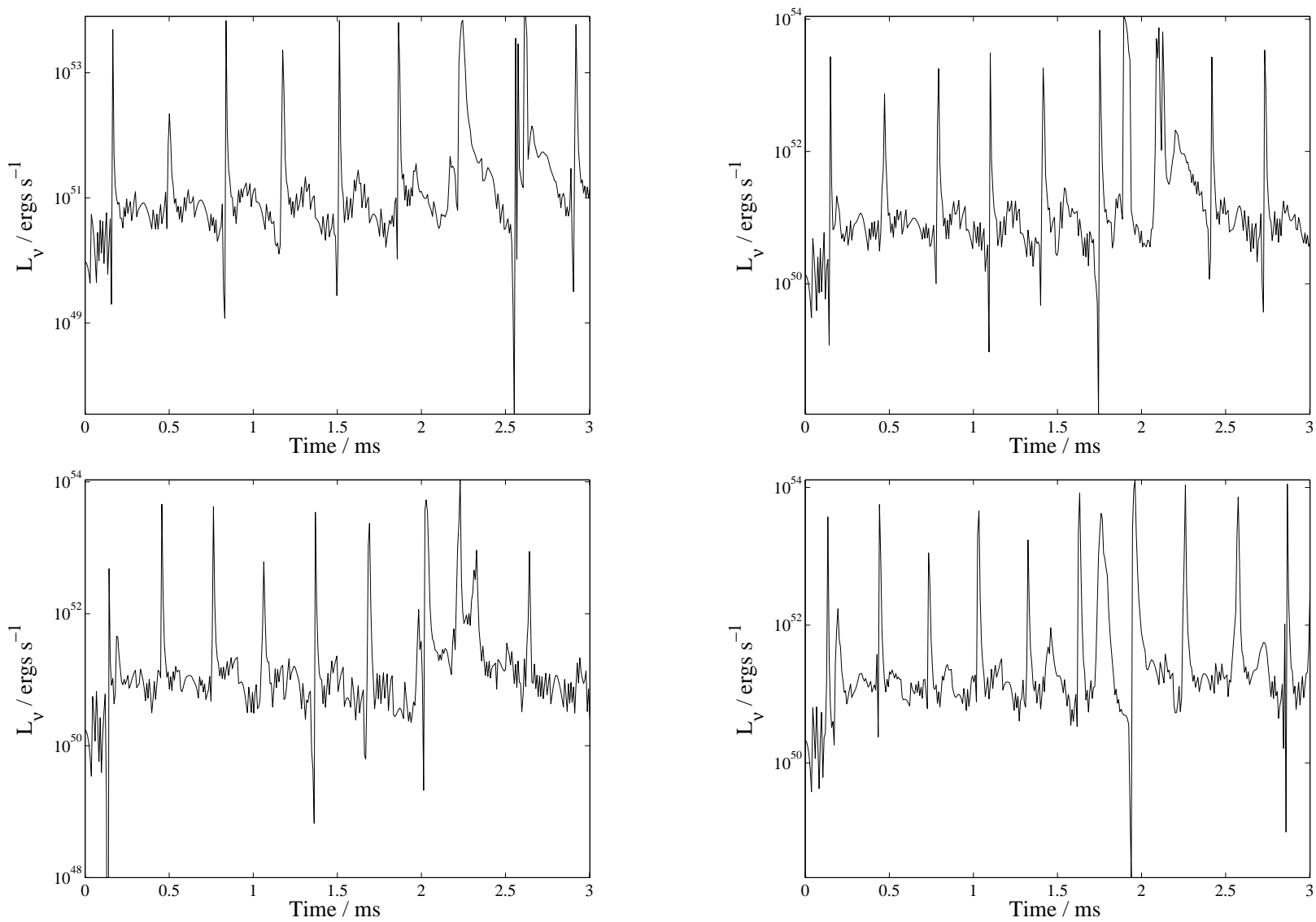

FIG. 8: Neutrino luminosity versus time for $M=1.55 M_{\odot}$, (left upper figure), $M=1.7 M_{\odot}$, (right upper figure), $M=1.8 M_{\odot}$, (left lower figure), $M=1.9 M_{\odot}$, (right lower figure).

The peak luminosities range from $10^{52}$ to $10^{54} \mathrm{ergs} / \mathrm{s}$; the pulsating period of the luminosity is the same as that of the temperature and of the density. 


\section{B. Pair Production Rate}

Neutrinos and antineutrinos can become electron and positron pairs via the neutrino and antineutrino annihilation process $\left(\nu+\bar{\nu} \rightarrow e^{-}+e^{+}\right)$. The total neutrino and antineutrino annihilation rate can be given as follows [57, 58]

$$
\begin{aligned}
\dot{Q}_{\nu \bar{\nu} \rightarrow e^{ \pm}} & =\frac{7 G_{F}^{2} \pi^{3} \zeta(5)}{2 c^{5} h^{6}} D\left[k T_{\nu}(t)\right]^{9} \int_{R_{\nu}}^{\infty} \Theta(r) 4 \pi r^{2} d r \\
& =\frac{7 G_{F}^{2} D \pi^{3} \zeta(5)}{2 c^{5} h^{6}} \frac{8 \pi^{3}}{9} R_{\nu}^{3}\left(k T_{\nu}\right)^{9}
\end{aligned}
$$

where $\Theta(r)=2 \pi^{2}(1-x)^{4}\left(x^{2}+4 x+5\right) / 3, x=\sqrt{1-R_{\nu}^{2} / r^{2}}, T_{\nu}(t)$ is the temperature at the neutrinosphere at time $t, G_{F}^{2}=5.29 \times 10^{-44}$ is the Fermi constant, $\zeta$ is the Riemann zeta function, and $D$ is a numerical value depending on the pair creation processes (e.g. experimental results indicate that $D_{1}=1.23$ for $\nu_{e} \nu_{\bar{e}}$ and $D_{2}=0.814$ for $\nu_{\mu} \nu_{\bar{\mu}}$ and $\nu_{\tau} \nu_{\bar{\tau}}$ ). To obtain the total neutrino annihilation rate from all species, $\nu_{e} \nu_{\bar{e}}, \nu_{\mu} \nu_{\bar{\mu}}$ and $\nu_{\tau} \nu_{\bar{\tau}}$, we sum up the energy rate for each single flavor,

$$
\begin{aligned}
\dot{Q} & =\dot{Q}_{\nu_{e} \bar{\nu}_{e}}+\dot{Q}_{\nu_{\mu} \bar{\nu}_{\mu}}+\dot{Q}_{\nu_{\tau} \bar{\nu}_{\tau}} \\
& =\frac{28 G_{F}^{2} \pi^{6} \zeta(5)}{9 c^{5} h^{6}}\left(D_{1}+2 D_{2}\right) R_{\nu}^{3}\left(k T_{\nu}\right)^{9}
\end{aligned}
$$

Fig. 9 shows the rate of energy carried away by the electron/positron pairs produced through neutrino annihilation, which varies from $\sim 10^{51} \mathrm{ergs} / \mathrm{s}$ to $\sim 10^{53} \mathrm{ergs} / \mathrm{s}$.

It is interesting to note that almost all neutrinos can be annihilated into electron-positron pairs at the peak because of the extremely high density and high energy of the neutrinos. In particular the rest mass of the electrons/positrons is much smaller than $k T_{\nu}$.

\section{MASS EJECTION AND ACCELERATION}

In order to calculate the mass ejected from the stellar surface by neutrinos/antineutrinos and pairs, a very detailed knowledge of the mass distribution near the surface is required. It was pointed out that the density profile in the crust plays a very important role in determining how much mass can be ejected [59]. They use a static star model and an assumed simple power law density profile to demonstrate that the mass ejection can be significantly different. In our computer capability the minimum spacial grid size that can be achieved is $0.28 \mathrm{~km}$. In order to estimate a precise location we choose to use the Piecewise 

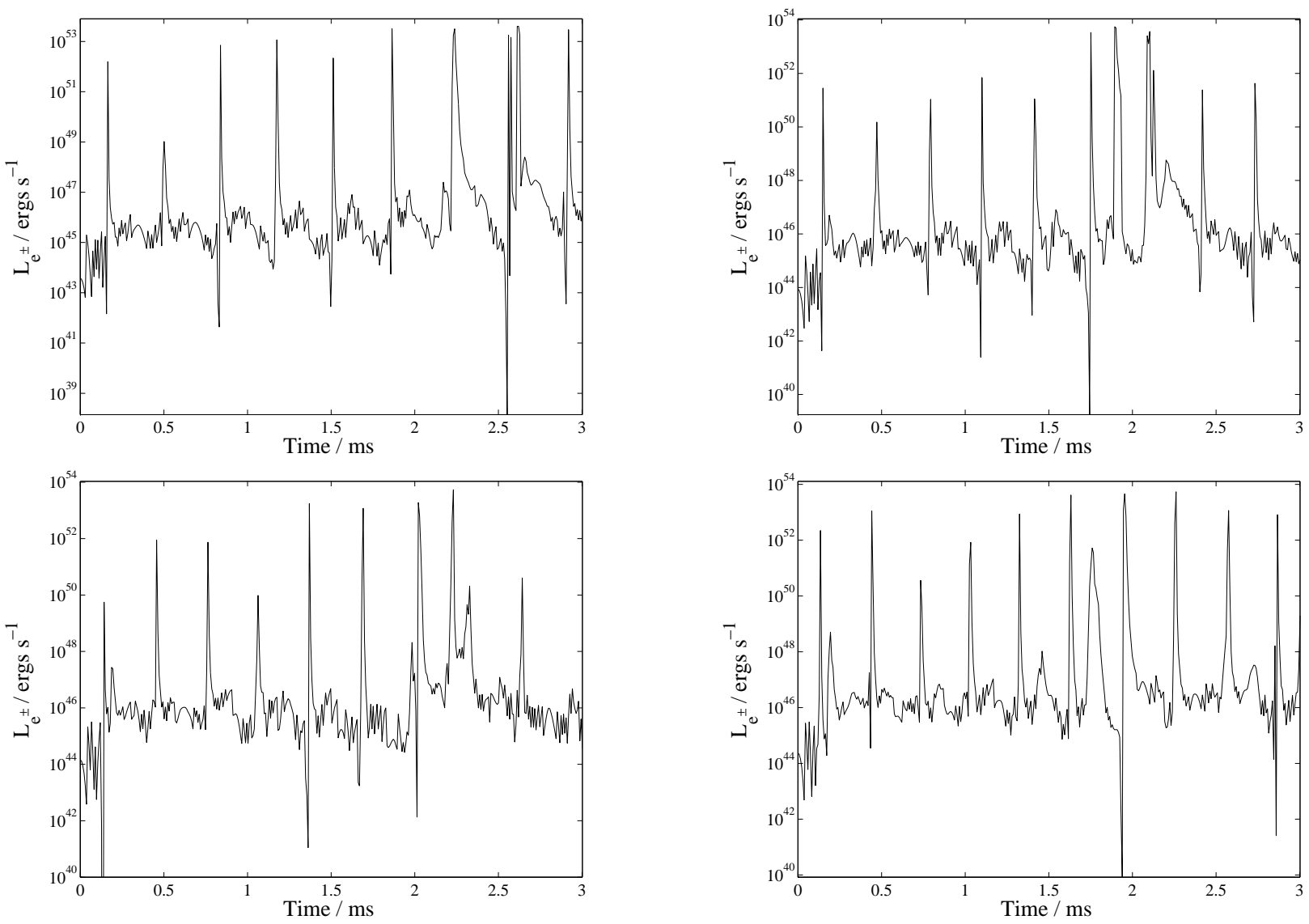

FIG. 9: Electron-positron luminosity versus time for $M=1.55 M_{\odot}$, (left upper figure), $M=1.7 M_{\odot}$, (right upper figure), $M=1.8 M_{\odot}$, (left lower figure), $M=1.9 M_{\odot},($ right lower figure).

Cubic Hermite Interpolating Polynomial (PCHIP) method to interpolate the density and the temperature data along the grids. The PCHIP method can provide a more accurate representation of the physical reality [60]. As compared with other interpolation methods (e.g., cubic spline data interpolation), the curve produced by the PCHIP method does not contain extraneous "bumps" or "wiggles", meaning that it could preserve the shape of the density and of the temperature profile, even when they change dramatically. However, it is unavoidable that even if we choose the best possible method, the true location might be slightly different from the real one. In Fig. 10 we compare the original time evolution of the position of the neutrinosphere $\left(R_{\nu}\right)$, temperature at $R_{\nu}$, density at $R_{\nu}$, and the neutrino luminosity with the results obtained by using the PCHIP method.

We can see that these quantities are qualitatively the same. We believe that the real continuous mass distributions near the stellar surface can be approximated by a continuous 

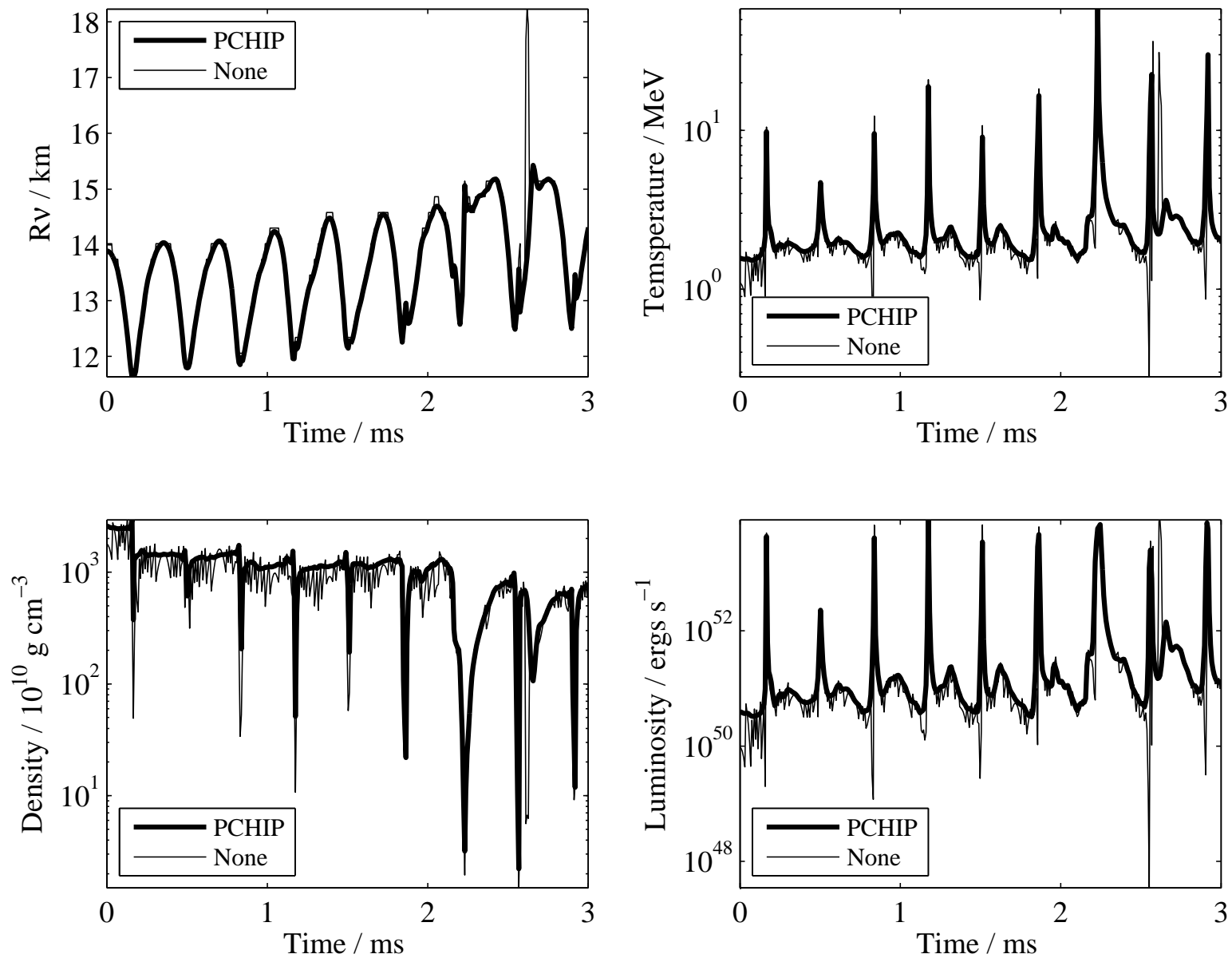

FIG. 10: Results with PCHIP method and without data interpolation for $\mathrm{M}=1.55 M_{\odot}$.

mass distribution function, obtained from the numerical simulated data by using a PCHIP method.

The mass ejection from a newly born quark stars was calculated in [59]. They argue that neutrino-electron scattering is the most dominated process to deposit the neutrino energy in the crust. In this paper we argue that the dominated energy deposition process is the neutrino-antineutrino annihilation process. It should be noted that the optical depth $(\tau)$, which is defined as $\tau=n \sigma l$, is the most important quantity to determine the energy deposition instead of cross section $(\sigma)$ alone. Here $n$ is the scattered particle density and $l$ is the characteristic length. We can only eject mass above neutrinosphere, which is located only several grid sizes below the stellar surface when the neutrino luminosity is maximum. From Fig. 4 we can see that the density is several $10^{11} \mathrm{~g} / \mathrm{cm}^{3}$ when the neutrino luminosity is maximum, where most of the mass is ejected. The density has dropped a factor of 10 in one 
grid size from the neutrinosphere to the stellar surface. Therefore the scale length of density is about half of grid size, i.e. $l \sim 0.14 \mathrm{~km}$. In a neutron rich matter, we can take electron fraction as 0.2 then we obtain $n_{e} l \sim 10^{39} \mathrm{~cm}^{-2}$. On the other hand, the neutrino density is uniform from the neutrinosphere to the surface of the star and the density of neutrino is given by $n_{\nu}=11 a T^{4} / 4 k T \sim 10^{36}(k T / 15 \mathrm{MeV})^{3}$, where $a$ is the Stefan-Boltzmann constant and $k$ is the Boltzmann constant. At the neutrino luminosity maximum, $k T \sim 15 \mathrm{MeV}$ and the distance from the neutrinosphere to the stellar surface is several grid sizes, which is $\sim 1 \mathrm{~km}$. Then $n_{\nu} l \sim 10^{41} \mathrm{~cm}^{-2}$. Since neutrino-antineutrino annihilation cross section (cf. Eq. 2 in [61]) and neutrino-electron scattering cross section (cf. Eq. 7 in [59]) are almost the same so we will ignore this process in calculating the mass ejection.

\section{A. Energy deposition in the crust and mass ejection}

Although most of the neutrinos/antineutrinos created above the neutrinosphere can escape, part of them can still be absorbed in the crust. We can estimate the amount of neutrino energy $E_{\nu}$ deposited in the crust due to the absorption in the following way. If we define $R_{\mathrm{M}}$ as $R_{\mathrm{NS}}>R_{\mathrm{M}}>R_{\nu}$, then the absorbed neutrino energy onto the surface mass layer between $R_{\mathrm{M}}$ and $R_{\mathrm{NS}}$ could be expressed as

$$
E_{\nu}\left(R_{\mathrm{M}}\right)=\int\left[1-e^{-\tau\left(R_{\mathrm{M}}\right)}\right] L\left(R_{\mathrm{M}}\right) d t
$$

where $\tau\left(R_{\mathrm{M}}\right)=\int_{R_{\mathrm{M}}}^{\infty} d r \kappa_{\text {eff }}(r)$ is the optical depth at $R_{\mathrm{M}}, L\left(R_{\mathrm{M}}\right)=21 \pi R_{\mathrm{M}}^{2} a c T\left(R_{\mathrm{M}}\right)^{4} / 8$ is the neutrino luminosity above $R_{\mathrm{M}}$ and $T\left(R_{\mathrm{M}}\right)$ is the temperature at $R_{\mathrm{M}}$. Notice that we only performed data output for every 20 iterations in our simulations, corresponding to a time interval $\Delta T=0.0075 \mathrm{~ms}$. Hence, the time interval $d t$ in the integral is taken to be $d t=\Delta T$.

The annihilated pairs created in the crust will be absorbed because of the much stronger interaction matter than that of neutrinos, and the pair energy $\left(E_{l^{ \pm}}\right)$deposited in the crust is given by

$$
\begin{gathered}
E_{l^{ \pm}}=\int \dot{Q}\left(R_{\mathrm{M}}\right) d t \\
\dot{Q}\left(R_{\mathrm{M}}\right)=\frac{7 G_{F}^{2} \pi^{3} \zeta(5)}{2 c^{5} h^{6}}\left(D_{1}+2 D_{2}\right)\left(k T_{\nu}\right)^{9} \int_{R_{\mathrm{M}}}^{R_{\mathrm{NS}}} \Theta(r) 4 \pi r^{2} d r .
\end{gathered}
$$

Note that we only integrate $r$ from $R_{\mathrm{M}}$ to $R_{\mathrm{NS}}$ instead of integrating from $R_{\nu}$ to $\infty$. 
The gravitational binding energy of the surface mass is

$$
E_{\mathrm{G}}=\frac{G M \Delta m}{R_{\mathrm{M}}},
$$

where $M=4 \pi \int_{0}^{R_{\mathrm{M}}} r^{2} \rho(r) d r$, and $\Delta m\left(R_{\mathrm{M}}\right)=4 \pi \int_{R_{\mathrm{M}}}^{R_{\mathrm{NS}}} r^{2} \rho(r) d r$, respectively.

Since the neutrino and pair absorption inside the neutron star actually happen simultaneously, we combine the absorbed neutrino and pair energy together to be $E^{\text {absorbed }}$

$$
E^{\text {absorbed }}=E_{l^{ \pm}}+E_{\nu} .
$$

As long as $E^{\text {absorbed }}\left(R_{\mathrm{M}}\right)>E_{\mathrm{G}}\left(R_{\mathrm{M}}\right)$, the surface layer of $\Delta m\left(R_{\mathrm{M}}\right)$ could be ejected from the neutron star. Hence from this criteria we can obtain the maximum ejected mass.

\section{B. Acceleration by pairs}

As we have mentioned in Section 4.2, the neutrino and antineutrino annihilation is very high at the peak of the neutrino pulses due to the extremely high density and the high energy of neutrinos. In fact most pairs are created outside the star, and therefore after the matter is ejected, it will be accelerated by absorbing the pairs created by the annihilation

processes. The annihilation energy created from the neutron star surface $R_{N S}$ to $r>R_{N S}$ is given by $E_{l^{ \pm}}=\int \dot{Q}_{\nu \bar{\nu} \rightarrow e^{ \pm}}(r, t) d t$, where

$$
\dot{Q}(r, t)=\frac{7 G_{F}^{2} \pi^{3} \zeta(5)}{2 c^{5} h^{6}}\left(D_{1}+2 D_{2}\right)\left[k T_{\nu}(t)\right]^{9} \int_{R_{N S}}^{r} \Theta\left(r^{\prime}\right) 4 \pi r^{\prime 2} d r^{\prime} .
$$

In the following we will briefly describe how the pairs accelerate the ejected matter. Before we present our calculations, we first describe the continuous mass ejection processes. The time slice interval of our output data is $\Delta T=0.0075 \mathrm{~ms}$. We can calculate the maximum amount of ejected mass only time slice by time slice.

At $T_{1}$ (Fig. 11 upper left), a layer of mass $\Delta M\left(R_{M}\right)$ is ejected when $E^{\text {absorbed }}\left(R_{M}\right)>$ $E_{\mathrm{G}}\left(R_{M}\right)$. The outer surface $R_{M 1 f}$ of the ejected mass is approximately $R_{N S}$, and the velocity of the mass at the outer surface is almost $c$; the inner surface of the ejected mass is $R_{M 1 s}$, where the velocity of the mass at the inner surface is the escaping velocity, which is almost half of the speed of light.

$T_{2}$ is the next time step when another layer of mass could be ejected. Before $T_{2}$ (Fig. 11 upper right), when the mass layer ejected at $T_{1}$ is flying outwards from the star at $T_{1}+t<T_{2}$, 

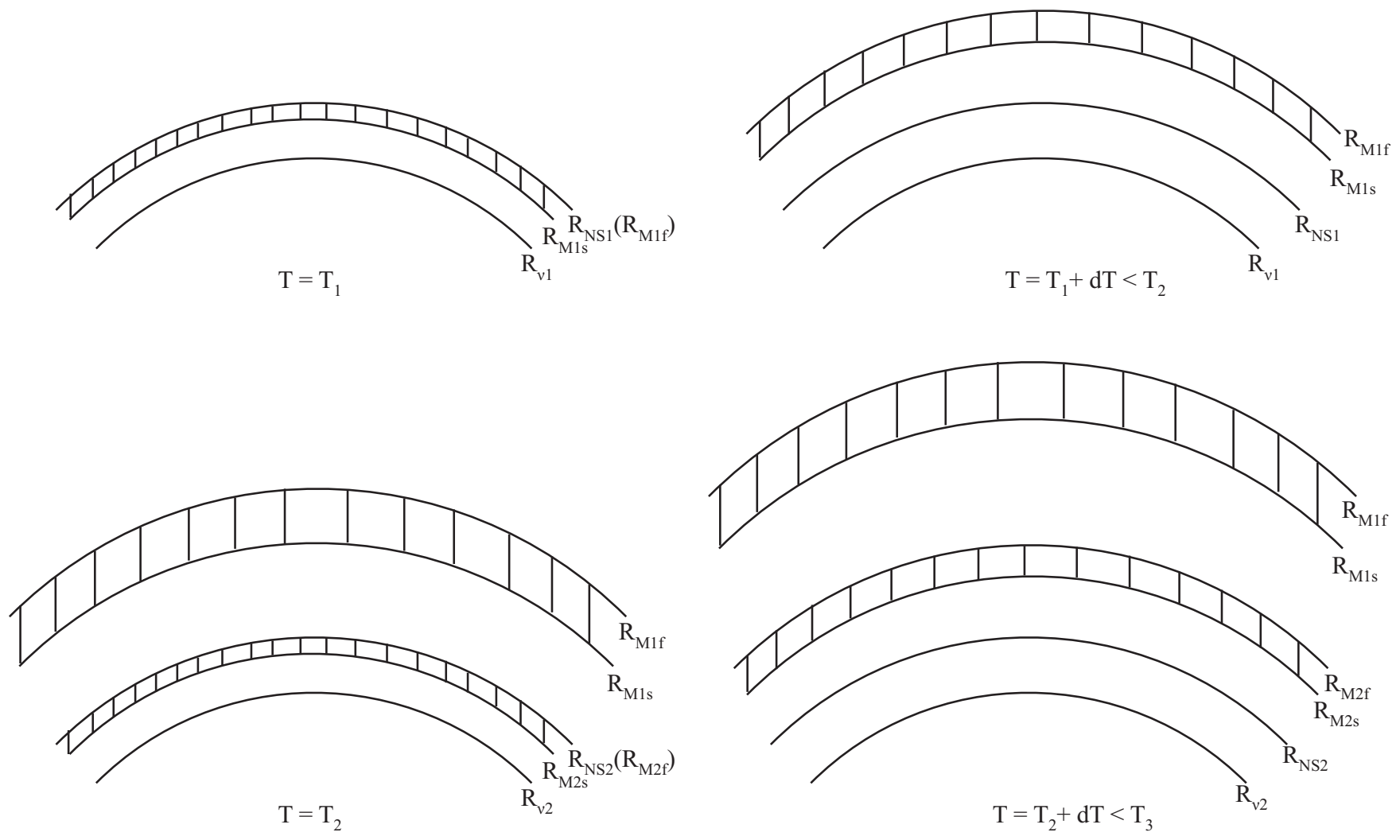

FIG. 11: Schematic illustration of mass ejection from the stellar surface.

the distance to the ejecta can be approximated as $R_{N S 1}+c t$ and $t$ is the elapsing time counted from $T_{1}$. In the space between $R_{N S 1}$ and $R_{N S 1}+c t$, pairs are created and they can move faster than the ejected mass layer. Eventually they are absorbed by the ejecta and accelerates the mass layer in front.

Another mass layer is ejected at $T_{2}$ (Fig. 11 lower left). When this ejecta moves outward, it will absorb pairs created between the stellar surface and this ejecta. However pairs created in the space between these two ejecta can still be absorbed by the first ejecta (cf. Fig. 11 lower right). The total pair energy for accelerating the mass layer ejected at $T_{1}$ is

$$
E^{\text {pair }}=\sum_{T_{i}=T_{1}}^{T_{\infty}} \int_{T_{i}}^{T_{i}+\Delta T} \dot{Q}_{T_{i}}\left(r_{1}, r_{2}\right) d t
$$

where

$$
\dot{Q}_{T_{i}}\left(r_{1}, r_{2}\right)=\frac{7 G_{F}^{2} \pi^{3} \zeta(5)}{2 c^{5} h^{6}}\left(D_{1}+2 D_{2}\right)\left(k T_{\nu}\right)^{9} \int_{r_{1}}^{r_{2}} \Theta(r) 4 \pi r^{2} d r
$$

is always calculated between the stellar surface and the ejecta or between two ejecta. 


\begin{tabular}{|c|c|c|c|c|}
\hline Pulse & $\Gamma$ & Energy $(\mathrm{ergs})$ & Mass $(\mathrm{g})$ & Time $(\mathrm{s})$ \\
\hline 1 & 17 & $2.2 \times 10^{46}$ & $1.5 \times 10^{24}$ & 0.16 \\
2 & $6.4 \times 10^{4}$ & $6.5 \times 10^{45}$ & $1.1 \times 10^{20}$ & 0.50 \\
3 & 1.6 & $4.7 \times 10^{48}$ & $9.1 \times 10^{27}$ & 0.83 \\
4 & 1.6 & $3.7 \times 10^{49}$ & $7.1 \times 10^{28}$ & 1.18 \\
5 & $1 \times 10^{6}$ & $4.2 \times 10^{46}$ & $4.6 \times 10^{19}$ & 1.50 \\
5 & $1.2 \times 10^{3}$ & $2.7 \times 10^{48}$ & $2.4 \times 10^{24}$ & 1.51 \\
6 & $2.8 \times 10^{4}$ & $4.4 \times 10^{48}$ & $1.7 \times 10^{23}$ & 1.85 \\
6 & 1.4 & $3.9 \times 10^{49}$ & $1.1 \times 10^{29}$ & 1.86 \\
7 & $3.8 \times 10^{2}$ & $4.4 \times 10^{51}$ & $1.3 \times 10^{28}$ & 2.21 \\
7 & 1.2 & $2.4 \times 10^{49}$ & $1.6 \times 10^{29}$ & 2.23 \\
8 & 1.2 & $8.9 \times 10^{48}$ & $5.0 \times 10^{28}$ & 2.55 \\
9 & 1.3 & $3.7 \times 10^{48}$ & $1.3 \times 10^{28}$ & 2.62 \\
10 & $2.5 \times 10^{5}$ & $2.3 \times 10^{48}$ & $1.1 \times 10^{22}$ & 2.90 \\
10 & 8.7 & $2.6 \times 10^{50}$ & $3.8 \times 10^{28}$ & 2.91 \\
\hline
\end{tabular}

TABLE I: Properties of the ejected mass. Ejected mass in some pulses are divided into two part: first part with sufficient mass and move faster; the later part moves slower and cannot collide with first part. $\left(M=1.55 M_{\odot}, \Gamma_{n}=1.85\right.$ and $B^{1 / 4}=160$ $\mathrm{MeV})$.

The Lorentz factor of each ejecta can be estimated as

$$
\Gamma=\frac{E^{\text {pair }}+E^{\text {absorbed }}}{m c^{2}}+1
$$

We summarize the results of the ejected masses in Tables 1-4. We can see that sometimes more than one layer of mass can be ejected in one pulse.

\section{POSSIBLE CONNECTION WITH GAMMA-RAY BURSTS}

\section{A. Duration of mass ejection}

We have pointed out that our numerical simulations are quite limited by the unphysical numerical damping. However, in realistic situations, the oscillations triggered by the collapse 


\begin{tabular}{|c|c|c|c|c|}
\hline Pulse & $\Gamma$ & Energy $(\mathrm{ergs})$ & Mass $(\mathrm{g})$ & Time $(\mathrm{s})$ \\
\hline 1 & 21 & $1.6 \times 10^{45}$ & $8.7 \times 10^{22}$ & 0.15 \\
2 & $2.6 \times 10^{2}$ & $1.0 \times 10^{45}$ & $4.4 \times 10^{21}$ & 0.47 \\
3 & $3.4 \times 10^{2}$ & $3.2 \times 10^{45}$ & $1.1 \times 10^{22}$ & 0.79 \\
4 & $1.3 \times 10^{2}$ & $5.3 \times 10^{45}$ & $4.5 \times 10^{22}$ & 1.10 \\
5 & $2 \times 10^{4}$ & $3.6 \times 10^{48}$ & $2.0 \times 10^{23}$ & 1.41 \\
6 & 2.2 & $6.9 \times 10^{49}$ & $6.2 \times 10^{28}$ & 1.74 \\
7 & 4.8 & $2.0 \times 10^{50}$ & $6.0 \times 10^{28}$ & 1.89 \\
8 & $2.3 \times 10^{2}$ & $3.1 \times 10^{48}$ & $1.5 \times 10^{25}$ & 1.91 \\
9 & $1.6 \times 10^{6}$ & $2.9 \times 10^{48}$ & $2.0 \times 10^{21}$ & 2.08 \\
9 & 5 & $4.7 \times 10^{50}$ & $1.3 \times 10^{29}$ & 2.09 \\
10 & 1.1 & $4.2 \times 10^{48}$ & $3.4 \times 10^{28}$ & 2.13 \\
11 & 8 & $1.2 \times 10^{46}$ & $1.9 \times 10^{24}$ & 2.41 \\
12 & 31 & $2.5 \times 10^{44}$ & $9.2 \times 10^{21}$ & 2.73 \\
\hline
\end{tabular}

TABLE II: Properties of the ejected mass. $\left(M=1.7 M_{\odot}, \Gamma_{n}=1.85\right.$ and $B^{1 / 4}=160$ $\mathrm{MeV})$.

must also be damped by some physical dissipation mechanisms. In this section, we would like to estimate how long the oscillations could last.

First, we note that hydrodynamics effects (e.g., shock waves, mass-shedding etc.) which can be modeled by our simulations certainly would play a role in the damping [17, 18]. In particular, the study of [18] suggests that the damping timescale due to hydrodynamics effects seen in their simulations is typically a few tens to hundreds ms. Another damping effect which exists in the case of rotational collapse is that due to gravitational radiation back-reaction. However, the damping timescale of this process is much longer than that of the hydrodynamics effects. The gravitational radiation damping is negligible and is in fact not taken into account in the works of [17, 18]. There are still other damping mechanisms. It was first pointed out in [62] that the dissipation due to nonleptonic reaction is of great importance and the stellar pulsations of the quark stars would be strongly damped via $s+u \leftrightarrow u+d$ in a few milliseconds. On the other hand, it was shown in (author?) [63] and [64] that in the high-temperature limit, which is exactly our case, the bulk viscosity is 


\begin{tabular}{|c|c|c|c|c|}
\hline Pulse & $\Gamma$ & Energy $(\mathrm{ergs})$ & Mass $(\mathrm{g})$ & Time $(\mathrm{s})$ \\
\hline 1 & $6.1 \times 10^{5}$ & $9.1 \times 10^{45}$ & $1.7 \times 10^{19}$ & 0.14 \\
2 & 30 & $2.5 \times 10^{46}$ & $9.5 \times 10^{23}$ & 0.46 \\
3 & 7.8 & $1.3 \times 10^{46}$ & $2.1 \times 10^{24}$ & 0.76 \\
4 & $7.9 \times 10^{4}$ & $1.6 \times 10^{47}$ & $2.2 \times 10^{21}$ & 1.06 \\
5 & 5.9 & $4.1 \times 10^{47}$ & $9.3 \times 10^{25}$ & 1.37 \\
6 & 14 & $3.5 \times 10^{48}$ & $3.1 \times 10^{26}$ & 1.68 \\
7 & $2.2 \times 10^{6}$ & $2.5 \times 10^{48}$ & $1.3 \times 10^{21}$ & 2.01 \\
7 & 8.2 & $4.2 \times 10^{50}$ & $6.4 \times 10^{28}$ & 2.01 \\
8 & $3.1 \times 10^{6}$ & $4.2 \times 10^{48}$ & $1.5 \times 10^{21}$ & 2.22 \\
8 & 3.9 & $1.9 \times 10^{50}$ & $7.4 \times 10^{28}$ & 2.22 \\
9 & $1.5 \times 10^{2}$ & $1.0 \times 10^{45}$ & $7.3 \times 10^{21}$ & 2.31 \\
10 & 59 & $7.3 \times 10^{43}$ & $1.4 \times 10^{21}$ & 2.64 \\
\hline
\end{tabular}

TABLE III: Properties of the ejected mass. $\left(M=1.8 M_{\odot}, \Gamma_{n}=1.85\right.$ and $B^{1 / 4}=160$ $\mathrm{MeV})$.

dramatically reduced. If we take $m_{s} \sim 140 \mathrm{MeV},\langle\rho\rangle \sim 10^{15} \mathrm{~g} / \mathrm{cm}^{3}$ and $\langle T\rangle \sim 50 \mathrm{MeV}$, the damping time scale is $\sim 10 \mathrm{~s}[63]$.

If all of the above physical mechanisms cannot efficiently damp out the oscillations, then the pulse neutrino emission and the produced mass ejection considered in this paper would be the only processes to damp out the oscillations. In Tables 1-4 we can see that the energy carried away by the ejecta in first $3 \mathrm{~ms}$ is of the order of $\sim 10^{50}$ ergs. The escaped neutrinos will carry away comparable amount of energy. The total oscillation energy is of the order of $\Delta E_{G} \sim G M^{2} \Delta R / R^{2}$, where $\Delta R$ is the change of radius before and after the phase transition. For the models presented in this paper, $\Delta R / R$ is of the order of $10 \%$, which gives oscillation energy of the order of several $10^{52}$ ergs. In other words the oscillations cannot last longer than several hundreds milliseconds even the neutrino emission and mass ejection are the only mechanisms to damp out the oscillations. 


\begin{tabular}{|c|c|c|c|c|}
\hline Pulse & $\Gamma$ & Energy $(\mathrm{ergs})$ & Mass $(\mathrm{g})$ & Time $(\mathrm{s})$ \\
\hline 1 & $2.6 \times 10^{2}$ & $6.1 \times 10^{46}$ & $2.6 \times 10^{23}$ & 0.13 \\
2 & 6.4 & $2.5 \times 10^{47}$ & $5.2 \times 10^{25}$ & 0.43 \\
3 & $6.3 \times 10^{4}$ & $1.4 \times 10^{46}$ & $2.4 \times 10^{20}$ & 0.73 \\
4 & $1 \times 10^{2}$ & $2.4 \times 10^{47}$ & $2.7 \times 10^{24}$ & 1.03 \\
5 & 1.42 & $1.9 \times 10^{49}$ & $4.9 \times 10^{28}$ & 1.32 \\
6 & $4.3 \times 10^{2}$ & $4.9 \times 10^{48}$ & $1.3 \times 10^{25}$ & 1.62 \\
6 & 2.2 & $7.0 \times 10^{49}$ & $6.5 \times 10^{28}$ & 1.63 \\
7 & $1 \times 10^{5}$ & $3.6 \times 10^{49}$ & $4.0 \times 10^{23}$ & 1.74 \\
8 & $9.6 \times 10^{4}$ & $5.2 \times 10^{48}$ & $6.0 \times 10^{22}$ & 1.94 \\
8 & 76 & $3.7 \times 10^{51}$ & $5.6 \times 10^{28}$ & 1.95 \\
9 & $8.8 \times 10^{3}$ & $5.7 \times 10^{48}$ & $7.2 \times 10^{23}$ & 2.25 \\
9 & 2.5 & $1.9 \times 10^{50}$ & $1.4 \times 10^{29}$ & 2.25 \\
10 & 7.5 & $2.7 \times 10^{47}$ & $4.6 \times 10^{25}$ & 2.55 \\
11 & 1.2 & $3.9 \times 10^{48}$ & $2.0 \times 10^{28}$ & 2.86 \\
\hline
\end{tabular}

TABLE IV: Properties of the ejected mass. $\left(M=1.9 M_{\odot}, \Gamma_{n}=1.85\right.$ and $B^{1 / 4}=160$ $\mathrm{MeV})$.

\section{B. Short GRBs}

There are two kinds of GRBs, i.e., long GRBs with duration larger than $\sim 2$ s and short GRBs with duration less than $\sim 2 \mathrm{~s}$ [2, 3]. The isotropic $\gamma$-ray energy released by a short GRB is usually in the range of $10^{49}-10^{51} \mathrm{ergs}$, i.e., about two or three orders of magnitude less than that of long GRBs [65]. Assuming that no more than $\sim 10 \%$ of the kinetic energy can be converted to $\gamma$-ray radiation, then an amount of kinetic energy up to $10^{50}-10^{52}$ ergs should be produced by the central engine. An example that has a relatively large energy release is GRB 051221A [66]. The isotropic $\gamma$-ray energy is $1.5 \times 10^{51} \mathrm{ergs}$, and an isotropic kinetic energy of $8.4 \times 10^{51}$ ergs has been estimated for this event [66].

It is widely believed that long GRBs may originate from the collapse of massive stars [6], while short GRBs may be connected with the merger of binary compact stars [65, 67]. However, it is still possible that some GRBs may be produced by other mechanisms. 
For example, GRB 060614, a special nearby long GRB that is not associated with any supernovae, may be produced by an intermediate mass black hole that captured and tidally disrupted a star [68]. Another interesting kind of central engine mechanism involves the phase transition of normal neutron stars to strange stars [16, 69, 70, 71]. Since the phase transition may be processed in a detonative mode, the details in this process are still largely uncertain and need further investigation. Especially, numerical simulations are necessary to help to understand the process.

\section{Basic equations}

The observations of X-ray, optical and radio afterglows from some well-localized GRBs have proved their cosmological origin [10, 72, 73, 74, 75]. The so-called fireball model [11, 76, 77, 78, 79, 80, 81, 82] can basically explain the observational facts well, and thus it is strongly favored, and widely accepted today. In this model, the central engine gives birth to some energetic ejecta intermittently, like a geyser, producing a series of ultra-relativistic shells. The shells collide with each other at a radius of $R_{\text {in }}$ and produce strong internal shocks. The highly variable $\gamma$-ray emission in the main burst phase of GRBs should be produced by these internal shocks. After the main burst phase, the shells merge into one main shell and continue to expand outward. It sweeps up circum-burst medium, being decelerated and producing external shocks. The observed long-lasting and steadily decaying afterglows (with a much smoother light curve as compared with the $\gamma$-ray light curve) should be due to these external shocks.

After examining the numerical results of our simulations, we believe that the gravitational collapse of a neutron star induced by the phase transition from normal nuclear matter to quark matter can be an ideal mechanism for producing GRBs. In this Section, we give some detailed explanations.

Let us first consider the general case of the collision between two typical shells. Following the description of [83], we assume that the first shell is ejected with a mass of $M_{1}$, a bulk velocity of $\beta_{1}$ and a bulk Lorentz factor of $\gamma_{1}$. The second shell is assumed to be ejected after a time interval of $\Delta t$, with the mass, velocity and Lorentz factor being $M_{2}, \beta_{2}$, and $\gamma_{2}$, respectively. Here, $\gamma_{1}=\left(1-\beta_{1}^{2}\right)^{-1 / 2}, \gamma_{2}=\left(1-\beta_{2}^{2}\right)^{-1 / 2}$, and they satisfy $\gamma_{2}>\gamma_{1} \gg 1$. The distance between the two shells is then initially $c \Delta t$. Since the second shell moves faster, it 
will finally catch up with the first shell at a radius of [83],

$$
R_{\text {in }}=\frac{\beta_{1} \beta_{2}}{\beta_{2}-\beta_{1}} c \Delta t \approx \frac{c \Delta t}{\beta_{2}-\beta_{1}}=\frac{2 \gamma_{1}^{2} \gamma_{2}^{2}}{\gamma_{2}^{2}-\gamma_{1}^{2}} c \Delta t .
$$

This is the place where the internal shock appears and the GRB takes place. At this point, the total elapsed time measured in the static burster frame is

$$
t_{\mathrm{in}}=\frac{R_{\mathrm{in}}}{\beta_{1} c} \approx \frac{\Delta t}{\beta_{2}-\beta_{1}}=\frac{2 \gamma_{1}^{2} \gamma_{2}^{2}}{\gamma_{2}^{2}-\gamma_{1}^{2}} \Delta t
$$

However, due to relativistic effect, the observed elapsed time since the beginning of the phase transition is only $t_{\text {in }} /\left(2 \gamma_{1}^{2}\right) \approx \gamma_{2}^{2} \Delta t /\left(\gamma_{2}^{2}-\gamma_{1}^{2}\right)$.

After the collision, the two shells will merge into one single shell and move at a new bulk velocity of $\beta_{\text {bul }}$ (correspondingly, with the Lorentz factor $\gamma_{\text {bul }}$ ). During the process, a portion of the initial bulk kinetic energy will be dissipated as random internal energy. We denote the average Lorentz factor of the random internal energy as $\gamma_{\mathrm{i}}$. Since momentum and energy are conserved in the collision, we have [83],

$$
\begin{aligned}
M_{1} \gamma_{1} \beta_{1}+M_{2} \gamma_{2} \beta_{2} & =\left(M_{1}+M_{2}\right) \gamma_{\mathrm{i}} \gamma_{\mathrm{bul}} \beta_{\mathrm{bul}} \\
M_{1} \gamma_{1}+M_{2} \gamma_{2} & =\left(M_{1}+M_{2}\right) \gamma_{\mathrm{i}} \gamma_{\mathrm{bul}} .
\end{aligned}
$$

It is then easy to get the solution for $\beta_{\mathrm{bul}}, \gamma_{\mathrm{bul}}$ and $\gamma_{\mathrm{i}}$ as

$$
\begin{gathered}
\beta_{\text {bul }}=\frac{M_{1} \gamma_{1} \beta_{1}+M_{2} \gamma_{2} \beta_{2}}{M_{1} \gamma_{1}+M_{2} \gamma_{2}}, \\
\gamma_{\text {bul }}=\frac{M_{1} \gamma_{1}+M_{2} \gamma_{2}}{\sqrt{M_{1}^{2}+M_{2}^{2}+2 M_{1} M_{2} \gamma_{1} \gamma_{2}\left(1-\beta_{1} \beta_{2}\right)}} \approx \\
\sqrt{\frac{M_{1} \gamma_{1}+M_{2} \gamma_{2}}{M_{1} / \gamma_{1}+M_{2} / \gamma_{2}}}, \\
\gamma_{\mathrm{i}}=\frac{M_{1} \gamma_{1}+M_{2} \gamma_{2}}{\left(M_{1}+M_{2}\right) \gamma_{\mathrm{bul}}} \approx \frac{\sqrt{M_{1} \gamma_{1}+M_{2} \gamma_{2}} \cdot \sqrt{M_{1} / \gamma_{1}+M_{2} / \gamma_{2}}}{M_{1}+M_{2}} .
\end{gathered}
$$

The efficiency of transferring bulk kinetic energy into internal energy is

$$
\epsilon=\left(\gamma_{\mathrm{i}}-1\right) / \gamma_{\mathrm{i}}=1-\gamma_{\mathrm{i}}^{-1}
$$

When two shells collide, the emission from the shock-accelerated electrons will correspond to a single pulse in the light curve of the GRB. The rising time of the pulse is mainly 
determined by the time needed for the shocks to cross the two shells. Since the merged shell is moving toward us ultra-relativistically with a Lorentz factor of $\gamma_{\text {bul }}$, only a small part of the shell (with an opening angle of $\sim 1 / \gamma_{\text {bul }}$ ) will be seen by us. The decay time of the pulse is then mainly determined by the arrival time lag of a photon emitted at the angle of $\sim 1 / \gamma_{\text {bul }}$ as compared with the photon emitted simultaneously at the line of sight. Usually, the decay time is longer than the rising time, so we can use the decay time to characterize the width of the pulse, i.e. [84, 85]

$$
\tau_{\text {pulse }} \approx R_{\text {in }} /\left(2 \gamma_{\text {bul }}^{2} c\right)
$$

\section{GRBs resulting from phase transition?}

According to our simulations, at least more than 10 shells can be ejected during the phase transition of a neutron star in the first $3 \mathrm{~ms}$. Among these shells, a few will have isotropic energies larger than $10^{48}$ ergs and expand ultra-relativistically with Lorentz factors larger than $>100$ (cf. Tables 1-4). Note that although our simulations only last for about $3 \mathrm{~ms}$, the actual duration of the oscillation process may last for several hundreds milliseconds, as argued at the end of our Section 6.1 . During this period, tens or even hundreds of ultrarelativistic shells might be ejected, each with an energy larger than $\sim 10^{48}$ ergs. The total energy enclosed in these relativistic shells may be $10^{50}-10^{51}$ ergs. We suggest that the collision between these shells can give birth to a GRB.

To describe this process in a quantitative way, we first study the collision between two specific (but typical) shells. We assume $M_{1}=M_{2}, \gamma_{1}=300, \gamma_{2}=2 \gamma_{1}=600$. We further assume that the second shell is ejected after a time interval of $\Delta t=1 \mathrm{~ms}$. It is then straightforward to find that they will collide at a radius of $R_{\text {in }} \sim 8 \gamma_{1}^{2} c \Delta t / 3 \approx 7.2 \times 10^{12} \mathrm{~cm}$, and at the time of $t_{\text {in }} \sim 8 \gamma_{1}^{2} \Delta t / 3 \approx 240 \mathrm{~s}$. Note that due to relativistic effect, the observed elapsed time since the beginning of the phase transition is only $t_{\text {in }} /\left(2 \gamma_{1}^{2}\right) \sim 4 \Delta t / 3 \approx 1.3 \mathrm{~ms}$.

The merged shell will move at a Lorentz factor of $\gamma_{\text {bul }} \sim \sqrt{2} \gamma_{1} \approx 420$, with the co-moving internal energy characterized by $\gamma_{\mathrm{i}} \approx 3 / 2 \sqrt{2} \approx 1.06$. The efficiency of transferring the bulk kinetic energy into internal energy is $\epsilon=1-1 / 1.06 \approx 5.6 \%$. The pulse will have a width of $\tau_{\text {pulse }} \approx 2 \Delta t / 3 \approx 0.6 \mathrm{~ms}$.

In realistic cases, the shells are ejected with variable masses, variable Lorentz factors, and variable time intervals, respectively. The conditions then become very complicated. 
For example, if $\gamma_{2}$ is much larger than $\gamma_{1}\left(\gamma_{2} \gg \gamma_{1}\right.$, but we still assume $\left.M_{2}=M_{1}\right)$, then the solution can be expressed as $R_{\text {in }} \sim 2 \gamma_{1}^{2} c \Delta t, t_{\text {in }} \sim 2 \gamma_{1}^{2} \Delta t, \gamma_{\text {bul }}=\sqrt{\gamma_{1} \gamma_{2}}, \gamma_{\mathrm{i}}=\sqrt{\gamma_{2} / 4 \gamma_{1}}$, $\tau_{\text {pulse }}=\Delta t \gamma_{1} / \gamma_{2}$. In this case, the pulse width will be very small. On the other hand, if $\gamma_{2}$ is only slightly larger than $\gamma_{1}$ (still with $M_{2}=M_{1}$ ), then $\gamma_{\text {bul }} \sim \gamma_{1}, \gamma_{i} \sim 1, R_{\text {in }}$ and $t_{\text {in }}$ will be very large so that a pulse much wider than $\Delta t$ can be generated. However, in this case, the energy transfer efficiency will be extremely low $(\epsilon \ll 1)$ for this single pulse.

In the standard fireball model, it is generally believed that the total duration of a GRB reflects the active period of the central engine. In our simulations, the shells are ejected mainly in a few hundred milliseconds. So, this mechanism is most proper for explaining short GRBs. The duration may reasonably range between a few ms and several hundred ms if the neutrino emission and the mass ejection are the only processes to damp out the stellar oscillations. It is also possible for the duration to extent to more than $1 \mathrm{~s}$. For example, if we take $\gamma_{1}=350, \gamma_{2}=400$ and $\Delta t=300 \mathrm{~ms}$, then the observed elapsed time for this pulse will be larger than $t_{\text {in }} /\left(2 \gamma_{1}^{2}\right)=\gamma_{2}^{2} /\left(\gamma_{2}^{2}-\gamma_{1}^{2}\right) \Delta t \approx 4.3 \Delta t \sim 1.3$ s. Note that the total isotropic energy of the shells can be $10^{50}-10^{51} \mathrm{ergs}$, and this is also consistent with the energy requirement of most short GRBs. If some extent of anisotropy exists in the process (which is quite possible if the effects of strong magnetic field of the compact star are further included, see the discussion in the last paragraph of this section), then the energy release can even meet the requirement of those rigorous energetic events, such as GRB 051221A [66]. Also, as noted above, if two shells are ejected with similar Lorentz factors, they may collide at a very late time. We further notice from Tables $1-4$ that there are many high energy shells that moves at trans-relativistic speeds (with Lorentz factors significantly less than $\sim 10$ ). Late collisions can also be produced by these energetic shells when they finally catch up with the decelerating external shock (that produces GRB afterglow). Such late collisions may manifest themselves as flaring activities (emerged in the afterglow phase), as frequently observed 1000 - 10000 s after the trigger of GRBs [86, 87].

In our simulations, the shells are ejected periodically, with a period of $\sim 0.3 \mathrm{~ms}$. However, it is quite unlikely that we could observe any obvious periodicity in the $\gamma$-ray light curve. The reason is that the shells have different masses, velocities, and energies. The periodicity will then be most likely smeared out at the time of collision.

The energy releases in our simulations are basically isotropic. The reason is that we did not include the effects of rotation and macroscopical magnetic field in our calculations. When 
these effects are considered, the energy releases should show some features of anisotropy. This is an interesting point that needs to be investigated in further details.

\section{DISCUSSIONS}

In this paper we have studied the possible consequences of the phase-induced collapse of neutron stars to hybrid stars. We have found that both the density and the temperature inside the star will oscillate with the same period, but almost $180^{\circ}$ out of phase, which will result in the emission of intense pulsating neutrinos. The temperatures at the peaks of pulsating neutrino fluxes are 10-20 MeV, which are 2-3 times higher than non-oscillating case. Since the electron/positron pair creation rate sensitively depends on the temperature at neutrinosphere, the efficiency of pair creation increases dramatically. We want to point out that the intense pulse neutrino and pair luminosity can be maintained due to the oscillatory fluid motion, which can carry thermal energy directly from the stellar core to the surface. This process can replenish the energy loss of neutrino emission much quicker than the neutrino diffusion process. Part of neutrino energy, roughly (1-1/e), and pairs inside the star will be absorbed by the matter very near the stellar surface. When this amount of energy exceeds the gravitational binding energy of matter, some mass near the stellar surface will be ejected, and this mass will be further accelerated by absorbing pairs created from the neutrino and antineutrino annihilation processes outside the star. Unlike inside the star, the surface properties, e.g. position of the neutrinosphere, surface temperature etc., are very sensitive to the surface perturbation. The neutrino and pair luminosities can be varied from pulse to pulse. This results in the large variation of the Lorentz factor of the mass ejecta. The internal collisions among these mass ejecta may produce the short time variabilities of the Gamma-ray Bursts, which can be as short as submilliseconds. Although mass ejecta are ejected periodically, each ejecta can have different masses and Lorentz factors as explained before. Therefore, the intrinsic period could not be observed. Although we can only simulate the oscillating stars for a few millisecond, we can speculate that this may be a possible mechanism for short Gamma-ray Bursts based on the following reasons. [89] have estimated that the viscous damping time for such oscillating system is of order of $10 \mathrm{~s}$. By assuming that if neutrinos and pair emissions are the only damping mechanisms,

the pulsations can last less than $\sim 3 s$ [89], which is roughly the characteristic time scale of 
short Gamma-ray Bursts.

The phase-transition from a neutron star to a strange star was simulated in [88], with the conclusion that this process is most likely not a gamma-ray burst mechanism. They mimic the phase-transition by the arbitrary motion of a piston deep within the star, and they have found that the mechanic wave will eject $\sim 10^{-2} M_{\odot}$ baryons, which causes the baryon contamination for the gamma-ray bursts. In our simulations, we assume a sudden change of equation of state to mimic the phase-transition, and we use the Newtonian hydrodynamic code to study the response of the stellar interior, after such a sudden change of the EOS. In our simulations we find that the mass ejection by the motion of the fluid is very small. We estimate that the major mass ejection would result from the heating of neutrinos and pairs on the stellar crust, which is not modeled in the simulations. Our total energy output and total mass in ejecta are close to that of [88] (cf. Tables 1-4). However, the neutrino energy injection is pulsating, and hence the mass ejection is also pulsating. The masses of individual ejecta range from $\sim 10^{-9} M_{\odot}$ to $\sim 10^{-4} M_{\odot}$, with output energy in the range of $10^{48} \operatorname{ergs}$ to $10^{50}$ ergs. Therefore, some ejecta cannot be relativistic, and they cannot contribute to GRBs. However, there are still many relativistic ejecta in each simulation model, which can have Lorentz factors $>100$, and with a total energy of $\sim 10^{50}-10^{51}$ ergs. We conclude that this could be a possible mechanism for short GRBs.

Finally, we want to remark that our numerical simulations describe a spherically symmetric, non-rotating, and collapsing stellar object. Also the effect of the magnetic fields was not taken into account. Therefore, the radiation emission produced in this model is isotropic. However, a realistic neutron star should have finite angular momentum and strong magnetic field, and hence these two factors could produce asymmetric mass ejection. This effect will be considered in future work.

\section{Acknowledgements}

We thank M.C. Chu, Z.G. Dai, P. Haensel, T. Lu, K.B. Luk, V.V. Usov and K.W. $\mathrm{Wu}$ for useful discussion, and the anonymous referee for very useful suggestions. KSC and TH are supported by the GRF Grants of the Government of the Hong Kong SAR under HKU7013/06P and HKU7025/07P respectively. YHF is supported by the National Science Foundation of China (grant 10625313), and the National Basic Research Program of China 
(grant 2009CB824800). LML is supported by the Hong Kong Research Grants Council (Grant No. CUHK4018/07P) and the direct grant (Project IDs: 2060330) from the Chinese University of Hong Kong. The computations were performed on the Computational Grid of the Chinese University of Hong Kong and the High Performance Computing Cluster of the University of Hong Kong.

[1] P. Mészáros, Rept. Prog. Phys. 69 (2006) 2259

[2] B. Zhang, Chin. J. Astron. Astrophys. 7 (2007) 1

[3] C. Kouveliotou et al., Astrophys. J. 413 (1993) L101

[4] G. Ghirlanda, G. Ghisellini and A. Celotti, Astron. Astrophys. 422 (2004) L5

[5] A. Fruchter et al., Nature 441 (2006) 436

[6] S. E. Woosley, Astrophys. J. 405 (1993) 273

[7] B. Paczyński, Astrophys. J. 494 (1998) L45

[8] A. I. MacFadyen and S. E. Woosley, Astrophys. J. 524 (1999) 262

[9] S. E. Woosley and J. S. Bloom, Ann. Rev. Astr. \& Astrophys. 44 (2006) 507

[10] J. S. Bloom et al., Astrophys. J. 638 (2006) 354

[11] B. Paczyński, Astrophys. J. 308 (1986) L43

[12] M. Ruffert and H. T. Janka, Astron. Astrophys. 344 (1999) 573

[13] C. D. Dermer and A. Atoyan, Astrophys. J. 643 (2006) L13

[14] A. J. Levan et al. 2006, Month. Not. R. Ast. Soc. 368 (2006) L1

[15] A. King, E. Olsson and M. B. Davies, Month. Not. R. Ast. Soc. 374 (2007) L34

[16] K. S. Cheng and Z. G. Dai, Phys. Rev. Lett. 77 (1996) 1210

[17] L. M. Lin, K. S. Cheng, M. C. Chu and W. M. Suen, Astrophys. J 639 (2006) 382

[18] E. B. Abdikamalov, H. Dimmelmeier, L. Rezzolla, and J. C. Miller, Month. Not. R. Ast. Soc. $392(2009) 52$

[19] N. Itoh, Prog. Theor. Phys. 44 (1970) 291

[20] A. R. Bodmer, Phys. Rev. 4 (1971) 1601

[21] E. Witten, Phys. Rev. D30 (1984) 272

[22] C. Alcock, E. Farhi, and A. Olinto, Astrophys. J. 310 (1986) 261

[23] P. Haensel, J. L. Zdunik, and R. Schaeffer, Astron. Astrophys. 160 (1986) 121 
[24] A. Drago, A. Lavagno, and I. Parenti, Astrophys. J. 659 (2007) 1519

[25] Z. Berezhiani, I. Bombaci, A. Drago, F. Frontera, and A. Lavagno, Astrophys. J. 586 (2003) 1250

[26] R. Venugopalan and A. P. Vischer, Phys. Rev. E49 (1994) 5849

[27] J. I. Kapusta, A. P. Vischer, and R. Venugopalan, Phys. Rev. C51 (1995) 901

[28] N. K. Glendenning, Compact stars, Nuclear Physics, Particle Physics and General Relativity, (2000) Springer, New York

[29] M. L. Olesen and J. Madsen, Phys. Rev. D47 (1993) 2313

[30] M. L. Olesen and J. Madsen, Phys. Rev. D49 (1994) 2698

[31] K. S. Cheng, Z. G. Dai, and T. Lu, Int. J. Mod. Phys. D7 (1998) 139

[32] K. S. Cheng and Z. G. Dai, 1998, Phys. Rev. Lett. 80 (1998) 18

[33] K. S. Cheng, Z. G. Dai, D. M. Wei, and T. Lu, Science 280 (1998) 407

[34] T. Harko, K. S. Cheng, and P. S. Tang, Astrophys. J. 608 (2004) 945

[35] J. E. Horvath and O. G. Benvenuto, Phys. Lett. B 213 (1988) 516

[36] H. T. Cho, K. W. Ng, and A. D. Speliotopoulos, Phys. Lett. B326 (1994) 111

[37] K. Iida and K. Sato, Phys. Rev. C58 (1998) 2538

[38] P. L. Roe, 1981, J. Comput. Phys. 43 (1981) 357

[39] P. Collela and P. R. Woodward, 1984, J. Comput. Phys. 54 (1984) 174

[40] Tian, X. L., M.Phil. Thesis (2008) University of Hong Kong

[41] T. DeGrand, R. L. Jaffe, K. Johnson, and J. Kiskis, Phys. Rev. D12 (1975) 2060

[42] H. Satz, 1982, Phys. Lett. B113 (1982) 245

[43] F. M. Steffens, H. Holtmann, and A. W. Thomas, Phys. Lett. B358 (1995) 139

[44] P. Haensel, in Final Stages of Stellar Evolution, Ed. C. Match \& J. M. Hamenry (Les Ulis: EDP Sciences) (2003) 249

[45] K. S. Cheng and Z. G. Dai, 1998, Astrophys. J. 492 (1998) 281

[46] I. Bombaci, I. Parenti, and I. Vidana, Astrophys. J. 614 (2004) 314

[47] S. L. Shapiro and S. A. Teukolsky, Black Holes, White Dwarfs and Neutron Stars: The Physics of Compact Objects (1983) (John Wiley \& Sons)

[48] M. Jaroszynski, Astron. Astrophys. 305 (1996) 839

[49] A. B. Balantekin and H. Yuksel, New J. Phys. 7 (2005) 51

[50] L. Wolfenstein, 1978, Phys. Rev. D17 (1978) 2369 
[51] H.-T. Janka, 1995, Astropart. Phys. 3 (1995) 377

[52] H.-T. Janka, Astron. Astrophys. 368 (2001) 527

[53] M. Maltoni, T. Schwetz, M. Tortola, and J. W. F. Valle, New Journal of Physics 6 (2004) 122

[54] O. Mena, I. Mocioiu, and S. Razzaque, Phys. Rev. D75 (2007) 063003

[55] E. Kh. Akhmedov and V. Niro, Journal of High Energy Physics 12 (2008) 106

[56] G. Altarelli and D. Meloni, 2009, Nucl. Phys. B809 (2009) 158

[57] J. Goodman, A. Dar, and S. Nussinov, Astrophys. J. 314 (1987) L7

[58] J. Cooperstein, L. J. V. D. Horn, and E. Baron, Astrophys. J. 321 (1987) L129

[59] P. Keränen, R. Ouyed, and P. Jaikumar, Astrophys. J. 618 (2005) 485

[60] F. N. Fritsch and R. E. Carlson, SIAM Journal on Numerical Analysis 17 (1980) 238

[61] K. Asano and T. Fukuyama, Astrophys. J. 531 (2000) 949

[62] Q. D. Wang and T. Lu, 1984, Physics Letters B148 (1984) 211

[63] R. F. Sawyer, 1989, Phys. Rev. D39 (1989) 3804

[64] J. Madsen, Phys. Rev. D46 (1992) 3290

[65] E. Nakar, Phys. Rept. 442 (2007) 166

[66] A. M. Soderberg, E. Berger, M. Kasliwal et al., Astrophys. J. 650 (2006) 261

[67] D. Eichler, M. Livio, T. Piran, and D. N. Schramm, Nature 340 (1989) 126

[68] Y. Lu, Y. F. Huang, and S. N. Zhang, Astrophys. J. 684 (2008) 1330

[69] I. Bombaci and B. Datta, Astrophys. J. 530 (2000) L69

[70] X. Y. Wang, Z. G. Dai, T. Lu, D. M. Wei, and Y. F. Huang, 2000, Astron. Astrophys. 357 (2000) 543

[71] R. Ouyed, J. Dey and M. Dey, Astron. Astrophys. 390 (2002) L39

[72] E. Costa et al., Nature 387 (1997) 783

[73] D. A. Frail, S. R. Kulkarni, L. Nicastro, M. Feroci, and G. B. Taylor, Nature 389 (1997) 261

[74] T. J. Galama, R. A. M. J. Wijers, M. Bremer, P. J. Groot, R. G. Strom, C. Kouveliotou, and J. van Paradijs, Astrophys. J. 500 (1998) L97

[75] N. Gehrels et al., Nature 437 (2005) 851

[76] J. Goodman, 1986, Astrophys. J. 308 (1986) L47

[77] P. Mészáros and M. J. Rees, Astrophys. J. 397 (1992) 570

[78] M. J. Rees and P. Mészáros, Month. Not. R. Ast. Soc. 258 (1992) 41P

[79] M. J. Rees and P. Mészáros, Astrophys. J. 430 (1994) L93 
[80] J. I. Katz, Astrophys. J. 422 (1994) 248

[81] R. Sari, R. Narayan, and T. Piran, Astrophys. J. 473 (1996) 204

[82] R. Sari, T. Piran, and R. Narayan, 1998, Astrophys. J. 497 (1998) L17

[83] B. Paczyński and G. Xu, Astrophys. J. 427 (1994) 708

[84] S. Kobayashi, T. Piran, and R. Sari, Astrophys. J. 490 (1997) 92

[85] T. Piran, Phys. Repts. 314 (1999) 575

[86] A. Burrows, R. Walder, C. D. Ott, and E. Livne, 2005, in The Fate of the Most Massive Stars, ASP Conference Series, Edited by R. Humphreys and K. Stanek, San Francisco: Astronomical Society of the Pacific, 332 (2005) 358

[87] P. Romano et al., Astron. Astrophys. 450 (2006) 59

[88] C. L. Fryer and S. E. Woosley, Astrophys. J. 501 (1988) 780

[89] T.C. Chan, K.S. Cheng, T. Harko, H.K. Lau, L.M. Lin, W.M. Suen and X. L. Tian, Astrophys. J. 695 (2009) 732 